\title{
Fine-Tuning the Dipole Moment of Asymmetric Non-Fullerene Ac- ceptors Enabling Efficient and Stable Organic Solar Cells
}

Zihao Xia, ${ }^{\text {aa }}$ Jinsheng Zhang, ${ }^{\ddagger \mathrm{b}}$ Xiang Gao, ${ }^{\mathrm{a}}$ Wei Song, ${ }^{\mathrm{b}}$ Jinfeng Ge, ${ }^{\mathrm{b}}$ Lin Xie, ${ }^{\mathrm{b}}$ Xiaoli Zhang, ${ }^{\mathrm{d}}$ Zhitian Liu ${ }^{* a}$ and Ziyi Ge*bc

${ }^{a}$ Hubei Engineering Technology Research Center of Optoelectronic and New Energy Materials, School of Materials Science w Engineering, Wuhan Institute of Technology, Wuhan, 430205, P. R. China.E-mail: able.ztliu@ wit.edu.cn

${ }^{b}$ Ningbo Institute of Materials Technology and Engineering, Chinese Academy of Sciences, Ningbo, P. R. China. geziyi@nimte.ac.cn 'Center of Materials Science and Optoelectronics Engineering, University of Chinese Academy of Sciences, Beijing 100049, China dSchool of Materials Science and Engineering, Zhengzhou University, 450001 P. R. China 


\section{Materials and Characterization}

PM6, Y6, PDINO were obtained from Solarmer Materials Inc, the molecular weight and dispersity of PM6 are $36 \mathrm{kDa}$ and 2.5-2.7, respectively. ${ }^{1} \mathrm{H}$ spectra and ${ }^{13} \mathrm{C}$ spectra were measured on a Bruker DMX-400 Spectrometer with $\mathrm{CDCl} 3$ as a solvent and rimethylsilane (TMS) as the internal reference. The molecular weights of the materials were analyzed by Bruker Autoflex III TOF mass spectrometerand. UV-visible absorption spectra were measured on a Perkin-Elmer Lambda 950 spectrophotometer. Cyclic voltammetry (CV) measurements were obtained on GHI 660D. Atomic force microscopy (AFM) measurements were performed using a Veeco Dimension 3100V atomic force microscope. DFT calculations were performed by using Gaussian 09B at the B3LYP/6-31G (d, p) and the alkyl chain was simplified as methyl. The Current density-voltage $(J-V)$ characteristics curves of all device were measured in N2-filled glove box using Keithley 2440 source meter with AM 1.5G solar simulator (Newport-Oriel ${ }^{\circledR}$ Sol3A 450W). EQE measurements were conducted with the solar cell QE tester (QE-R, Enli Technology Co., Ltd). The thickness of active layers was measured by Step Profilometer Dektak 150 .

\section{Device fabrication and measurement}

OSCs were fabricated with conventional structure of ITO/PEDOT: PSS/Active Layer/PDINO/Al. The ITO glass substrates were precleaned sequentially by deionized water, acetone and isopropanol for 30 minutes. The ITO glass was treated with UV-Ozone for 30 minutes before use. The PEDOT: PSS solution was spin-coated at 3000 rpm onto the ITO glass substrates for $60 \mathrm{~s}$ then dried at $130 \square$ for 20 minutes in air. The donor: acceptor blends with weight ratio of 1:1.2 and total $18 \mathrm{mg} / \mathrm{ml}$ dissolved in chloroform with mixed of 1-chloronaphthalene $(0.5 \%, \mathrm{v} / \mathrm{v})$. The blend solution was spin-coated on the PEDOT: PSS layer at $3000 \mathrm{rpm}$ for $60 \mathrm{~s}$ after being stirred on a hotplate of $65 \square$ for 20 minutes. Then it was annealed at $120^{\circ} \mathrm{C} 5$ minutes for PTIC-4Cl and PT2IC-4Cl blend films, while $100{ }^{\circ} \mathrm{C} 10$ minutes for TB-4Cl blend film. A thin layer of PDINO was cast onto the active layer and the $100 \mathrm{~nm} \mathrm{Al}$ as cathode was evaporated in vacuum chamber $\left(3 \times 10^{-6} \mathrm{mbar}\right)$. 


\section{Space Charge Limited Current Measurement}

The charge transport properties were measured by the space charge limited current (SCLC) method. the structures of hole-only devices were fabricated with ITO/PEDOT: PSS/active layer/MoO3/Ag and the electron-only devices were fabricated by ITO/ZnO/active layer/ PDINO/Al. The mobilities of device were calculated by the equation: $J=9 \varepsilon_{o} \varepsilon_{r} \mu\left(V^{2} / 8 L^{3}\right)$, in which $J$ is the current density, $\varepsilon_{0}$ is the permittivity of free space, $\varepsilon_{\mathrm{r}}$ is the permittivity of the active layer, $\mu$ is the mobility of hole or electron and $\mathrm{L}$ is the thickness of the active layer, $V$ is the internal voltage in the device ( $V=V_{\text {app }}-V_{\mathrm{bi}}$ ), where $V_{\text {app }}$ is the applied voltage and $V_{\text {bi }}$ is the offset voltage.

\section{S1.1 Materials and Synthesis}

All solvents and reagents were purchased from commercial sources and directly used without further purification, the toluene was died by solvent purification system. All reactions were conducted without water and oxygen.
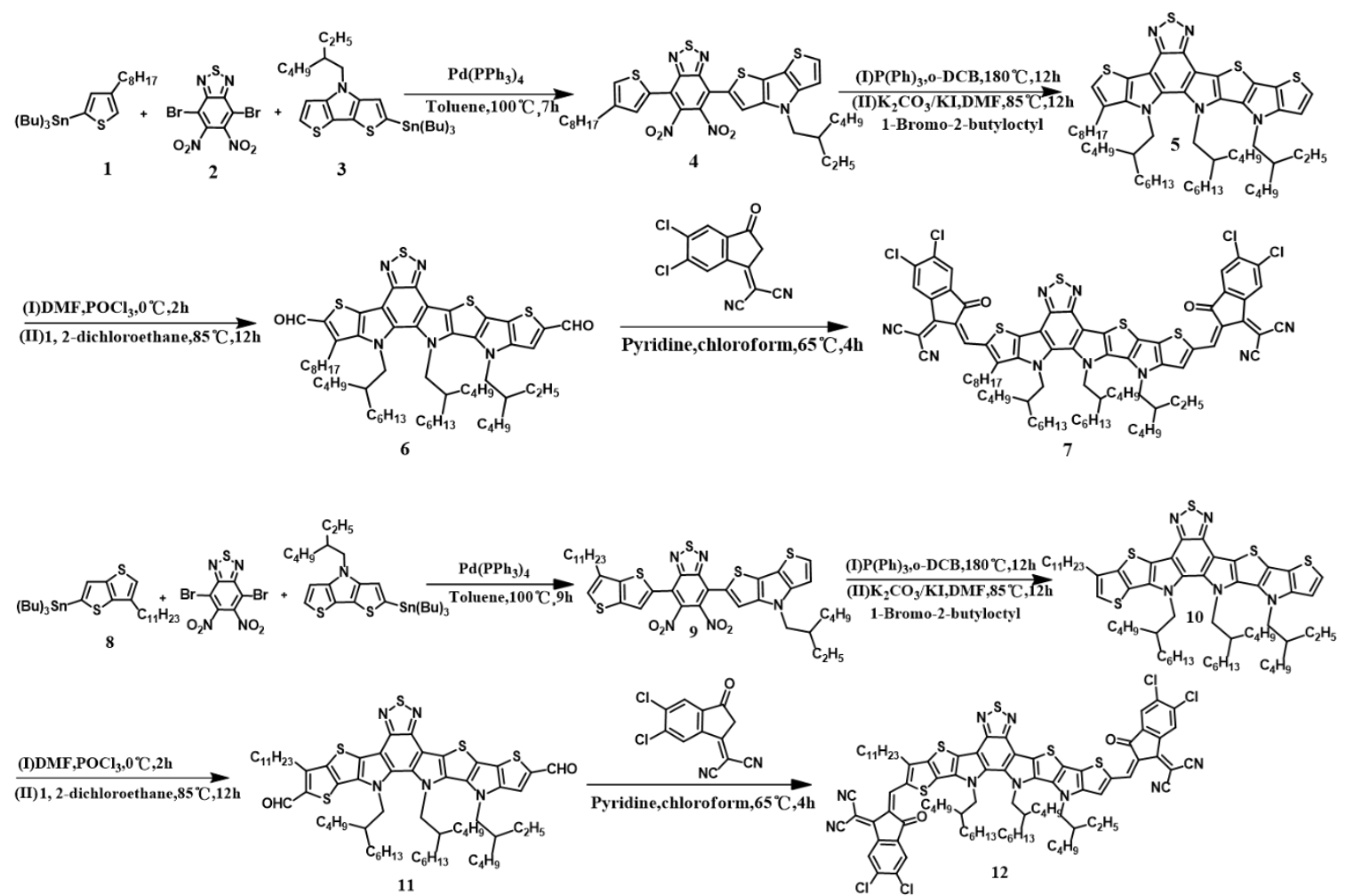

Scheme S1. Synthetic routes of PTIC-4Cl and PT2IC-4Cl. 


\section{Synthesis of 2-(5,6-dinitro-7-(4-octylthiophen-2-yl)benzo[c][1,2,5] thiadiazol-4-yl)-4-(2-ethylhexyl)-4H- dithieno[3,2-b:2',3'-d]pyrrole (compound 4)}

To a dry $250 \mathrm{~mL}$ round-bottom flask, compound1 $(4.00 \mathrm{~g}, 8.24 \mathrm{mmol})$, compound $2(2.64 \mathrm{~g}, 6.87 \mathrm{mmol})$ and compound 3 (4.78 g, $8.24 \mathrm{mmol})$ was dissolved in anhydrous toluene, then the $\mathrm{Pd}\left(\mathrm{PPh}_{3}\right)_{4}(396 \mathrm{mg}, 343.36 \mu \mathrm{mol})$ was added and the mixture was refluxed at $100 \square$ for 7 hours, after cooling to room temperature, the solvent was evaporated under vacuum and the crude product was purified by silica chromatography using petroleum/dichloromethane (2:1, v/v) as eluent to get a purple solid (1.2g, 31\%). ${ }^{1} \mathrm{H}$ NMR (400 MHz, Chloroformd) $\delta 7.46(\mathrm{~s}, 1 \mathrm{H}), 7.30(\mathrm{~s}, 2 \mathrm{H}), 7.00(\mathrm{~d}, \mathrm{~J}=5.3 \mathrm{~Hz}, 1 \mathrm{H}), 4.13-4.04(\mathrm{~m}, 2 \mathrm{H}), 2.66(\mathrm{t}, \mathrm{J}=7.7 \mathrm{~Hz}, 2 \mathrm{H}), 1.96-1.88(\mathrm{~m}$, $1 \mathrm{H}), 1.66(\mathrm{~m}, \mathrm{~J}=7.4 \mathrm{~Hz}, 2 \mathrm{H}), 1.41-1.21(\mathrm{~m}, 18 \mathrm{H}), 0.96-0.83(\mathrm{~m}, 9 \mathrm{H})$.

\section{Synthesis of 12,13-bis(2-butyloctyl)-11-(2-ethylhexyl)-1-octyl-12,13-dihydro-11H-[1,2,5]thiadiazolo-}

\section{$[3,4 \mathrm{e}]$ thieno $\left[2^{\prime \prime}, 3^{\prime \prime}: 4^{\prime}, 5^{\prime}\right]$ pyrrolo $\left[2^{\prime}, 3^{\prime}: 4,5\right]$ thieno[3,2b]thieno[2',3':4,5] pyrrolo[3,2-g]indole (compound 5)}

To a dry $100 \mathrm{~mL}$ round-bottom flask, compound $4(800 \mathrm{mg}, 1.13 \mathrm{mmol})$ and $\mathrm{PPh}_{3}(2.95 \mathrm{~g}, 11.3 \mathrm{mmol})$ was dissolved in extra dry o-dichlorobenzene (o-DCB, $15 \mathrm{~mL}$ ). After refluxing at $180{ }^{\circ} \mathrm{C}$ for 12 hours, the reaction was cooled to room temperature and the solvent was removed by silicon chromatography. The crude product was directly used without further purification for next step. potassium iodide $(102.79 \mathrm{mg}, 0.62 \mathrm{mmol})$, potassium carbonate (1.71 $\mathrm{g}, 12.38 \mathrm{mmol})$, 2- butyloctyl bromide $(1.85 \mathrm{mg}, 7.43 \mathrm{mmol})$ were dissolved in $\mathrm{N}, \mathrm{N}$-dimethylformamide $(15 \mathrm{ml})$. After stirring at $80{ }^{\circ} \mathrm{C}$ for 12 hours, the mixture was extracted with dichloromethane when it comes to room temperature, the crude product was purified by silica chromatography using petroleum/dichloromethane $(1: 2 \mathrm{v} / \mathrm{v})$ as eluent to get a yellow solid (320 mg, 40\%). ${ }^{1} \mathrm{H}$ NMR (400 MHz, Chloroform- $d$ ) $\delta 7.17(\mathrm{~d}, J=5.2 \mathrm{~Hz}, 1 \mathrm{H}), 7.07$ $(\mathrm{d}, J=5.6 \mathrm{~Hz}, 2 \mathrm{H}), 4.80-4.43(\mathrm{~m}, 6 \mathrm{H}), 2.92(\mathrm{q}, J=7.9 \mathrm{~Hz}, 2 \mathrm{H}), 2.05(\mathrm{p}, J=7.2 \mathrm{~Hz}, 2 \mathrm{H}), 1.92-1.80(\mathrm{~m}, 3 \mathrm{H}), 1.55$ $-1.11(\mathrm{~m}, 33 \mathrm{H}), 1.10-0.36(\mathrm{~m}, 44 \mathrm{H})$. 
Synthesis of 12,13-bis(2-butyloctyl)-11-(2-ethylhexyl)-1-octyl-12,13-dihydro -11H-[1,2,5]-thiadiazolo $[3,4 \mathrm{e}]-$ thieno[2",3':4',5']-pyrrolo[2',3':4,5]-thieno[3,2b]-thieno[2',3':4,5]-pyrrolo[3,2-g]-indole-2,9-

\section{dicarbaldehyde (compound 6)}

To a dry $50 \mathrm{~mL}$ two-necked round bottom flask, phosphorous oxychloride $(0.56 \mathrm{ml}, 6.11 \mathrm{mmol})$ and anhydrous $\mathrm{N}, \mathrm{N}$-dimethylformamide $(0.48 \mathrm{ml}, 6.11 \mathrm{mmol})$ were added gradually by syringe into the $10 \mathrm{ml}$ dry 1,2 dichloroethane, and the solution was cooled to $0^{\circ} \mathrm{C}$ and stirred for 2 hours, compound 5 (300 $\left.\mathrm{mg}, 0.30 \mathrm{mmol}\right)$ dissolved in another $10 \mathrm{ml}$ dry 1 , 2-dichloroethane was added to the solution, then the mixture was heated to $85{ }^{\circ} \mathrm{C}$ and stirred overnight. After cooling to room temperature, $100 \mathrm{ml}$ water was added to quench the reaction. The mixture was extracted with dichlorometane and the organic layer was washed with water and dried with anhydrous $\mathrm{Na}_{2} \mathrm{CO}_{3}$, after evaporating under reduced pressure, the crude product was purified by silica chromatography using petroleum/ ethyl acetate $(1 / 10, \mathrm{v} / \mathrm{v})$ to get compound $6(230 \mathrm{mg}, 73 \%)$ as a yellow solid. ${ }^{1} \mathrm{H}$ NMR (400 MHz, Chloroform-d) $\delta$ $10.14(\mathrm{~s}, 1 \mathrm{H}), 9.91(\mathrm{~s}, 1 \mathrm{H}), 7.70(\mathrm{~s}, 1 \mathrm{H}), 4.59-4.33(\mathrm{~m}, 6 \mathrm{H}), 3.60-3.51(\mathrm{~m}, 1 \mathrm{H}), 3.04(\mathrm{~s}, 1 \mathrm{H}), 2.19(\mathrm{~s}, 1 \mathrm{H}), 2.03-$ $1.75(\mathrm{~m}, 5 \mathrm{H}), 1.51-1.04(\mathrm{~m}, 37 \mathrm{H}), 0.96-0.10(\mathrm{~m}, 37 \mathrm{H})$.

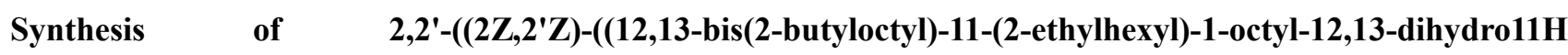
$[1,2,5]$ thiadiazolo $\left[3,4\right.$ e] thieno[2", $\left.3^{\prime \prime}: 4^{\prime}, 5^{\prime}\right]$ pyrrolo $\left[2^{\prime}, 3^{\prime}: 4,5\right]$ thieno[3,2b]thieno[2',3':4,5]pyrrolo[3,2-g]indole2,9-diyl)bis(methanylylidene))bis(5,6-dichloro-3-oxo-2,3-dihydro-1H-indene-2,1-diylidene)) dimalononitrile (compound 7)

To a dry $50 \mathrm{~mL}$ two-necked round bottom flask, compound 6 (200 mg, $0.19 \mathrm{mmol})$ and 2-(5,6-dichloro-3-oxo2,3-dihydro-1H-inden1-ylidene) malononitrile (2ClIC) (253 mg, $0.96 \mathrm{mmol}$ ) were dissolved in chloroform (20 mL), then pyridine $(1 \mathrm{ml})$ was added dropwise, the solution was heated to $65^{\circ} \mathrm{C}$ and stirred for 4 hours. After the reaction was completed, the reaction was poured into $200 \mathrm{~mL}$ of methanol and the crude product was purified by silica chromatography using dichloromethane/petroleum $(3 / 1, \mathrm{v} / \mathrm{v})$ to obtain a dark blue solid $(235 \mathrm{mg}, 80 \%) .{ }^{1} \mathrm{H}$ NMR (600 MHz, Chloroform-d) $\delta 9.22(\mathrm{~s}, 1 \mathrm{H}), 8.93(\mathrm{~s}, 1 \mathrm{H}), 8.80$ (s, 1H), $8.73(\mathrm{~s}, 1 \mathrm{H}), 7.98$ (d, J = 1.6 Hz, 1H), 7.90 (d, J $=2.6 \mathrm{~Hz}, 2 \mathrm{H}), 4.56(\mathrm{dq}, \mathrm{J}=24.7,13.3 \mathrm{~Hz}, 4 \mathrm{H}), 4.47-4.32(\mathrm{~m}, 2 \mathrm{H}), 3.55(\mathrm{~s}, 1 \mathrm{H})$, 
$3.16(\mathrm{~d}, \mathrm{~J}=8.4 \mathrm{~Hz}, 1 \mathrm{H}), 2.21(\mathrm{~s}, 1 \mathrm{H}), 2.04-1.81(\mathrm{~m}, 4 \mathrm{H}), 1.53-0.98(\mathrm{~m}, 33 \mathrm{H}), 0.94--0.09(\mathrm{~m}, 38 \mathrm{H}) .{ }^{13} \mathrm{C} \mathrm{NMR}$ (101 MHz, Chloroform-d) $\delta 185.84,159.32,158.44,147.73,147.13,144.97,143.58,140.13,139.62,139.26,139.16$, $139.13,138.89,138.77,138.69,136.75,136.15,136.06,135.95,132.06,131.38,126.77,126.70,125.80,125.12$, 124.82, 120.08, 115.46, 115.03, 114.77, 67.77, 56.11, 53.61, 53.22, 39.30, 39.23, 32.32, 31.96, 31.85, 31.66, 31.44, $31.12,30.02,29.94,29.73,29.59,29.47,29.42,29.31,29.06,28.83,28.25,28.06,27.96,23.19,22.80,22.48,22.38$, 22.29, 14.10, 14.08, 13.88, 11.02, 10.32. TOF MS: Calcd for $\mathrm{C}_{84} \mathrm{H}_{91} \mathrm{Cl}_{4} \mathrm{~N}_{9} \mathrm{O}_{2} \mathrm{~S}_{4}\left(\mathrm{M}^{+}\right)$:1528.75, Found: 1528.46 .

\section{Synthesis of 2-(5,6-dinitro-7-(6-undecylthieno[3,2-b]thiophen-2-yl)benzo[c][1,2,5] thiadiazol-4-yl)-4-(2- ethylhexyl)-4H-dithieno[3,2-b:2',3'-d]pyrrole (compound 9)}

To a dry $250 \mathrm{~mL}$ round-bottom flask, compound $8(4.00 \mathrm{~g}, 6.85 \mathrm{mmol})$, compound $2(2.19 \mathrm{~g}, 5.71 \mathrm{mmol})$ and compound 3 (3.98 g, $6.85 \mathrm{mmol}$ ) was dissolved in anhydrous toluene, then the $\mathrm{Pd}\left(\mathrm{PPh}_{3}\right)_{4}(330 \mathrm{mg}, 325.60 \mu \mathrm{mol})$ was added and the mixture was refluxed at $100 \square$ for 9 hours, after cooling to room temperature, the solvent was evaporated under vacuum and the crude product was purified by silica chromatography using petroleum/dichloromethane $(2: 1, \mathrm{v} / \mathrm{v})$ as eluent to get a purple solid $(1.33 \mathrm{~g}, 29 \%) .{ }^{1} \mathrm{H}$ NMR $(400 \mathrm{MHz}$, Chloroformd) $\delta 7.67(\mathrm{~d}, \mathrm{~J}=3.8 \mathrm{~Hz}, 1 \mathrm{H}), 7.48(\mathrm{~d}, \mathrm{~J}=3.7 \mathrm{~Hz}, 1 \mathrm{H}), 7.31(\mathrm{t}, \mathrm{J}=4.6 \mathrm{~Hz}, 1 \mathrm{H}), 7.16(\mathrm{~d}, \mathrm{~J}=3.7 \mathrm{~Hz}, 1 \mathrm{H}), 7.00(\mathrm{t}, \mathrm{J}=$ $4.5 \mathrm{~Hz}, 1 \mathrm{H}), 4.09(\mathrm{dt}, \mathrm{J}=7.4,3.7 \mathrm{~Hz}, 2 \mathrm{H}), 2.77(\mathrm{~m}, \mathrm{~J}=7.8,3.8 \mathrm{~Hz}, 2 \mathrm{H}), 1.97-1.72(\mathrm{~m}, 4 \mathrm{H}), 1.45-1.20(\mathrm{~m}, 27 \mathrm{H})$, $0.97-0.81(\mathrm{~m}, 10 \mathrm{H})$

\section{Synthesis of 13,14-bis(2-butyloctyl)-12-(2-ethylhexyl)-3-undecyl-13,14-dihydro-12H-[1,2,5]thiadiazolo $[3,4$ e] thieno[2",3":4',5']pyrrolo[2',3':4,5] thieno[3,2b]thieno[2",3"::4',5']thieno[2',3':4,5]pyrrolo[3,2- g]indole(compound 10)}

To a dry $100 \mathrm{~mL}$ round-bottom flask, compound $9(600 \mathrm{mg}, 0.74 \mathrm{mmol})$ and $\mathrm{PPh}_{3}(1.95 \mathrm{~g}, 7.40 \mathrm{mmol})$ was dissolved in extra dry o-dichlorobenzene (o-DCB, $15 \mathrm{~mL}$ ). After refluxing at $180 \square$ for 12 hours, the reaction was cooled to room temperature and the solvent was removed by silicon chromatography. The crude product was directly used without further purification for next step. potassium iodide $(66.92 \mathrm{mg}, 0.40 \mathrm{mmol})$, potassium carbonate $(1.11 \mathrm{~g}$, $8.06 \mathrm{mmol}), 2$ - butyloctyl bromide (1.21 mg, $4.84 \mathrm{mmol})$ were dissolved in $\mathrm{N}$, N-dimethylformamide (15 ml). After stirring at $80 \square$ for 12 hours, the mixture was extracted with dichloromethane when it comes to room temperature, the crude product was purified by silica chromatography using petroleum/dichloromethane $(1: 2 \mathrm{v} / \mathrm{v})$ as eluent to get a yellow solid (305mg, 35\%). ${ }^{1} \mathrm{H}$ NMR (400 MHz, Chloroform- $d$ ) $\delta 7.18(\mathrm{~d}, J=5.2 \mathrm{~Hz}, 1 \mathrm{H}), 7.07$ (d, $J=5.3 \mathrm{~Hz}$, $1 \mathrm{H}), 7.00(\mathrm{~d}, J=9.6 \mathrm{~Hz}, 1 \mathrm{H}), 4.71-4.57(\mathrm{~m}, 2 \mathrm{H}), 4.52(\mathrm{~d}, J=8.5 \mathrm{~Hz}, 4 \mathrm{H}), 2.83(\mathrm{t}, J=7.7 \mathrm{~Hz}, 2 \mathrm{H}), 2.08-1.94(\mathrm{~m}$, $5 \mathrm{H}), 1.86(\mathrm{p}$, 
$J=7.7 \mathrm{~Hz}, 3 \mathrm{H}), 1.50-1.05(\mathrm{~m}, 54 \mathrm{H}), 0.86(\mathrm{tq}, J=16.7,9.8,7.3 \mathrm{~Hz}, 21 \mathrm{H}), 0.55-0.09(\mathrm{~m}, 12 \mathrm{H})$.

Synthesis of 13,14-bis (2-butyloctyl)-12-(2-ethylhexyl)-3-undecyl-13,14-dihydro-12H-[1,2,5] thiadiazolo[3,4e]thieno[2", $\left.3^{\prime \prime}: 4^{\prime}, 5^{\prime}\right]$ pyrrolo $\left[2^{\prime}, 3^{\prime}: 4,5\right]$ thieno[3,2b] thieno $\left[2^{\prime \prime}, 3^{\prime \prime}: 4^{\prime}, 5^{\prime}\right]$ thieno[2',3':4,5]pyrrolo[3, 2-g]indole(compound 11)

To a dry $50 \mathrm{~mL}$ two-necked round bottom flask, phosphorous oxychloride $(0.34 \mathrm{ml}, 3.70 \mathrm{mmol})$ and anhydrous $\mathrm{N}, \mathrm{N}$-dimethylformamide $(0.29 \mathrm{ml}, 3.70 \mathrm{mmol})$ were added gradually by syringe into the $10 \mathrm{ml}$ dry 1,2 -dichloroethane, and the solution was cooled to $0 \square$ and stirred for 2 hours, compound 5 (200mg, $0.18 \mathrm{mmol}$ ) dissolved in another $10 \mathrm{ml}$ dry 1, 2-dichloroethane was added to the solution, then the mixture was heated to $85{ }^{\circ} \mathrm{C}$ and stirred overnight. After cooling to room temperature, $100 \mathrm{ml}$ water was added to quench the reaction. The mixture was extracted with dichlorometane and the organic layer was washed with water and dried with anhydrous $\mathrm{Na}_{2} \mathrm{CO}_{3}$. After evaporating under reduced pressure, the crude product was purified by silica chromatography using petroleum/ ethyl acetate $(1 / 10$, $\mathrm{v} / \mathrm{v})$ to get compound $6(157 \mathrm{mg}, 73 \%)$ as a yellow solid. ${ }^{1} \mathrm{H}$ NMR $(400 \mathrm{MHz}$, Chloroform- $d$ ) $\delta 10.14(\mathrm{~s}, 1 \mathrm{H}), 9.91$ (s, 1H), $7.70(\mathrm{~s}, 1 \mathrm{H}), 4.64(\mathrm{~d}, J=7.7 \mathrm{~Hz}, 2 \mathrm{H}), 4.55-4.25(\mathrm{~m}, 4 \mathrm{H}), 3.20(\mathrm{t}, J=7.7 \mathrm{~Hz}, 2 \mathrm{H}), 2.13(\mathrm{~d}, J=51.0 \mathrm{~Hz}$, 2H), $1.93(\mathrm{p}, J=7.8 \mathrm{~Hz}, 3 \mathrm{H}), 1.50-1.02(\mathrm{~m}, 44 \mathrm{H}), 0.96-0.08(\mathrm{~m}, 40 \mathrm{H})$.

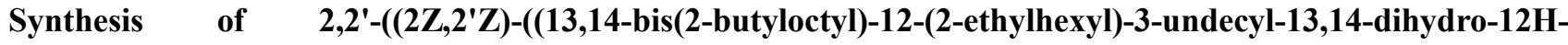
$[1,2,5]$ thiadiazolo[3,4-e]thieno[2",3":4',5']pyrrolo[2',3':4,5] thieno[3,2b]thieno[2",3":4',5']-thieno

[2',3':4,5]pyrrolo[3,2-g]indole-2,10-diyl)bis(methanylylidene))bis(5,6-dichloro-3-oxo-2,3-dihydro-1H-indene-

\section{2,1-diylidene))dimalononitrile(compound 12)}

To a dry $50 \mathrm{~mL}$ two-necked round bottom flask, compound 6 (200mg, $0.17 \mathrm{mmol})$ and 2-(5,6-dichloro-3-oxo2,3-dihydro-1H-inden1-ylidene) malononitrile (2ClIC) $(231 \mathrm{mg}, 0.88 \mathrm{mmol})$ were dissolved in chloroform (20 $\mathrm{mL})$, then pyridine $(1 \mathrm{ml})$ was added dropwise, the solution was heated to $65{ }^{\circ} \mathrm{C}$ and stirred for 4 hours. After the reaction was completed, the reaction was poured into $200 \mathrm{~mL}$ of methanol and the crude product was purified by silica chromatography using dichloromethane/petroleum (3/1, v/v) to obtain a dark blue solid $(230 \mathrm{mg}, 80 \%) .{ }^{1} \mathrm{H}$ NMR (600 MHz, Chloroform- $d$ ) $\delta 9.22(\mathrm{~s}, 1 \mathrm{H}), 8.94(\mathrm{~s}, 1 \mathrm{H}), 8.80(\mathrm{~s}, 1 \mathrm{H}), 8.72(\mathrm{~s}, 1 \mathrm{H}), 7.98(\mathrm{~s}, 1 \mathrm{H}), 7.89(\mathrm{~s}, 2 \mathrm{H})$. 
$4.58(\mathrm{~m}, J=21.1,20.1,10.1 \mathrm{~Hz}, 4 \mathrm{H}), 4.41(\mathrm{ddd}, J=44.2,15.2,8.0 \mathrm{~Hz}, 2 \mathrm{H}), 3.60-3.50(\mathrm{~m}, 1 \mathrm{H}), 3.15(\mathrm{~s}, 1 \mathrm{H}), 2.22$ (q, $J=7.2,6.8 \mathrm{~Hz}, 1 \mathrm{H}), 2.03-1.80(\mathrm{~m}, 4 \mathrm{H}), 1.52-1.10(\mathrm{~m}, 42 \mathrm{H}), 1.01-0.28(\mathrm{~m}, 40 \mathrm{H}), 0.08(\mathrm{~d}, J=16.0 \mathrm{~Hz}, 2 \mathrm{H})$. TOF MS: Calcd for $\mathrm{C}_{89} \mathrm{H}_{97} \mathrm{Cl}_{4} \mathrm{~N}_{9} \mathrm{O}_{2} \mathrm{~S}_{5}\left(\mathrm{M}^{+}\right): 1626.92$, Found: 1626.53 .

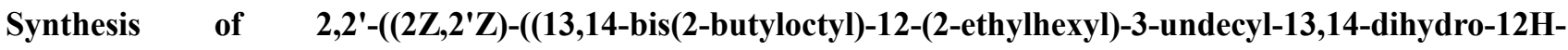
[1,2,5] thiadiazolo[3,4-e]thieno[2",3":4',5']pyrrolo[2',3':4,5] thieno[3,2-b]thieno[2",3":4',5']

thieno[2',3':4,5]pyrrolo[3,2-g]indole-2,10-diyl)bis(methanylylidene)) bis(5,6-dichloro-3-oxo-2,3-dihydro-1Hindene-2,1-diylidene))dimalononitrile(TB-4CI).

The final product of $\mathrm{TB}-4 \mathrm{Cl}$ was synthesized according to the previous reported methods of our work. ${ }^{1} \mathrm{H}$ NMR $(600 \mathrm{MHz}$, Chloroform- $d) \delta 9.23(\mathrm{~s}, 1 \mathrm{H}), 9.18(\mathrm{~s}, 1 \mathrm{H}), 8.80(\mathrm{~d}, J=7.5 \mathrm{~Hz}, 2 \mathrm{H}), 7.97(\mathrm{~d}, J=5.1 \mathrm{~Hz}, 2 \mathrm{H}), 4.81-4.44$ (m, 6H), $3.22(\mathrm{t}, J=8.0 \mathrm{~Hz}, 3 \mathrm{H}), 2.07-1.67(\mathrm{~m}, 8 \mathrm{H}), 1.50(\mathrm{~d}, J=17.3 \mathrm{~Hz}, 7 \mathrm{H}), 1.39-0.20(\mathrm{~m}, 56 \mathrm{H}) .{ }^{13} \mathrm{C} \mathrm{NMR}$ (101 MHz, Chloroform-d) $\delta 186.08,185.61,159.40,158.73,153.73,153.00,147.62,145.66,145.31,143.66,140.12$, 138.20, 135.73, 135.61, 135.46, 135.25, 133.58, 133.45, 130.83, 120.47, 120.41, 115.26, 114.98, 114.83, 114.72, $112.63,112.44,112.16,69.21,68.20,55.10,54.43,40.74,40.62,32.29,31.91,31.83,31.18,29.83,29.66,29.39$, 29.25, 29.17, 28.12, 22.79, 22.64, 14.10, 13.69. TOF MS: Calcd for $\mathrm{C}_{74} \mathrm{H}_{74} \mathrm{Cl}_{4} \mathrm{~N}_{9} \mathrm{O}_{2} \mathrm{~S}_{4}\left(\mathrm{M}^{+}\right)$:1377.50, Found: 1377.38. 
(a)

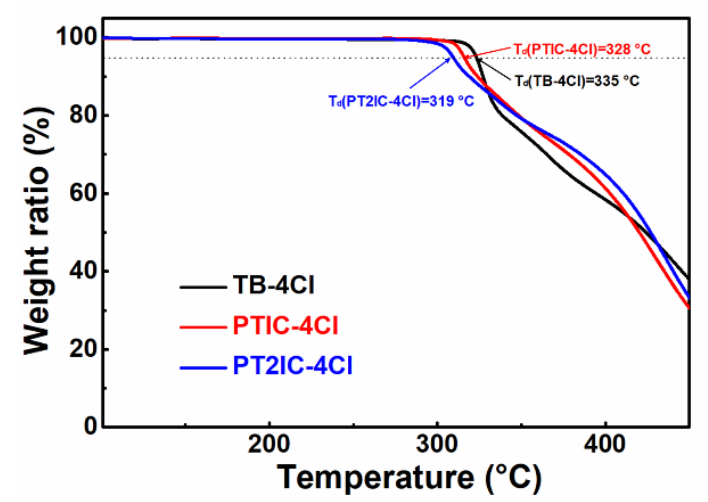

(b)

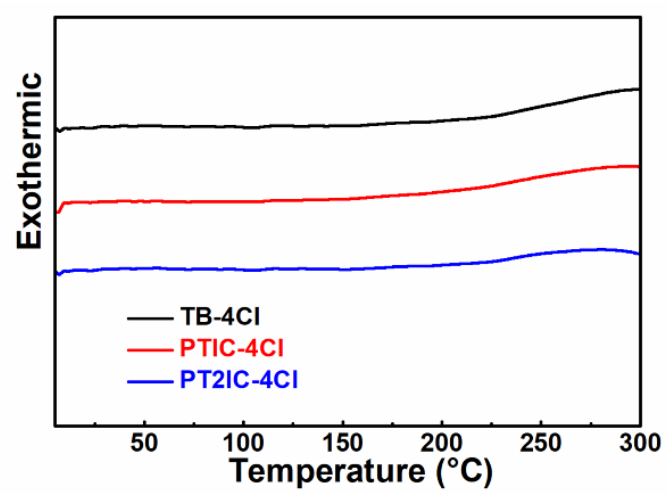

Figure S1. (a) TGA curves of TB-4Cl, PTIC-4Cl, PT2IC-4Cl with a heating rate of $10{ }^{\circ} \mathrm{C} \mathrm{min}-1$ under nitrogen; (b) Second heating DSC traces of TB-4Cl, PTIC-4Cl, PT2IC-4Cl with a heating rate of $10^{\circ} \mathrm{C} \mathrm{min}^{-1}$ under nitrogen.

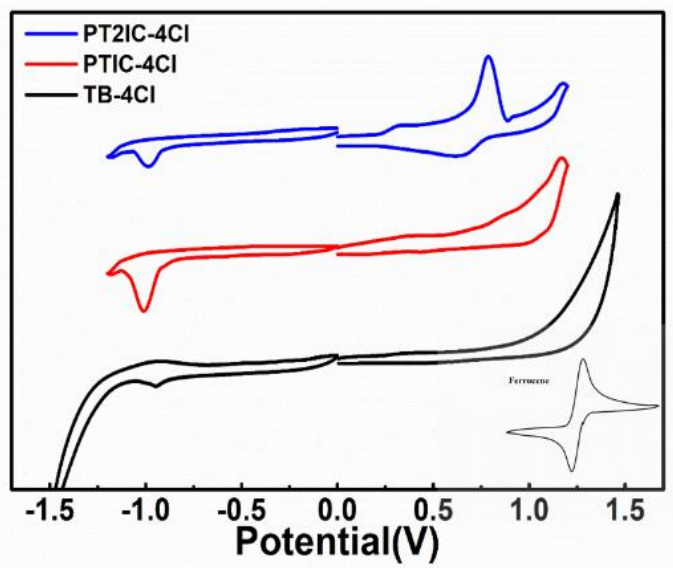

Figure S2. CV measurements of TB-4Cl, PTIC-4Cl and PT2IC-4Cl.

(a)

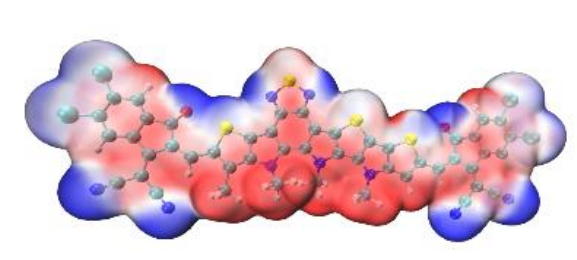

(b)

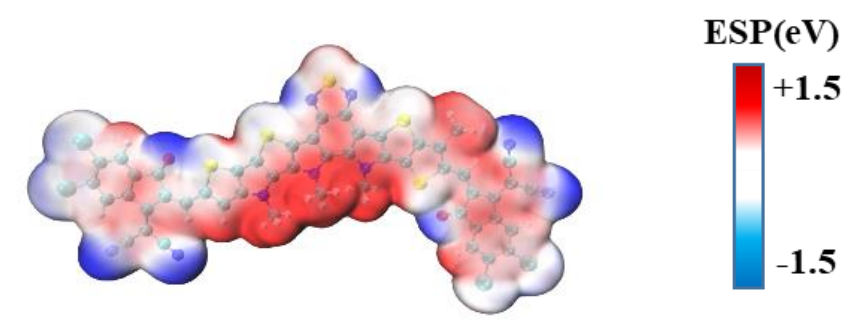

Figure S3. The ESP distribution of (a) PTIC-4Cl and (b) PT2IC-4Cl. 
(a)

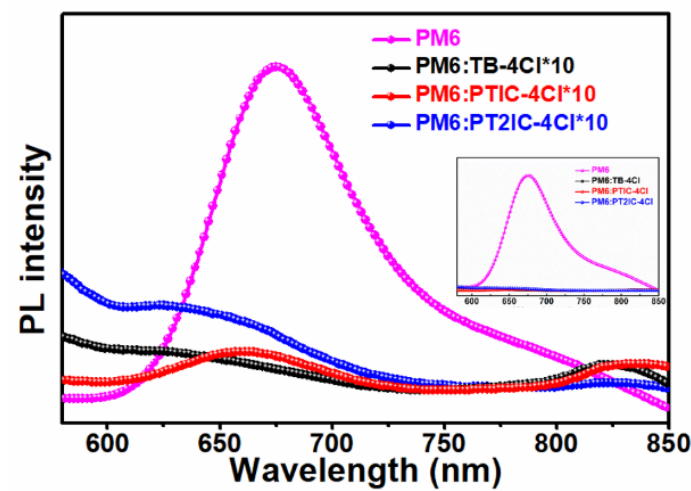

(c)

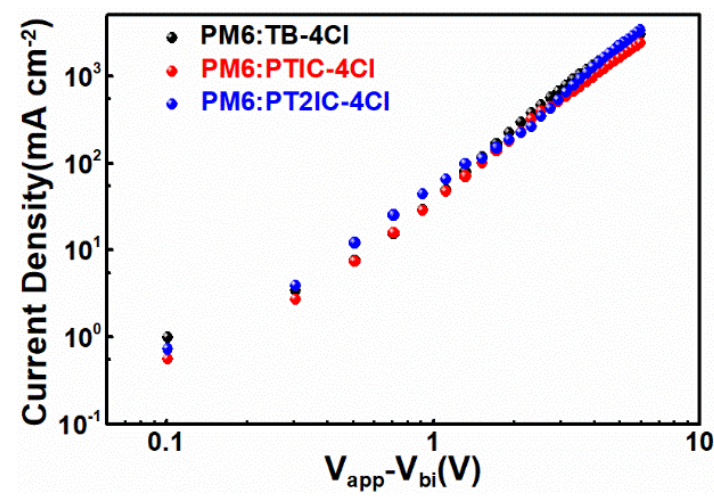

(b)

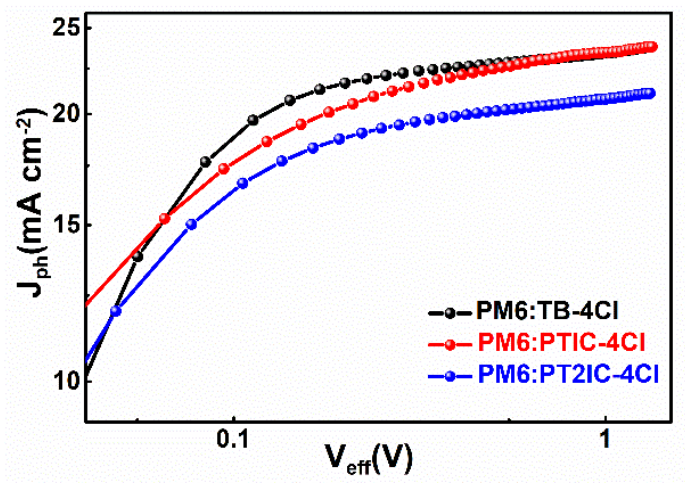

(d)

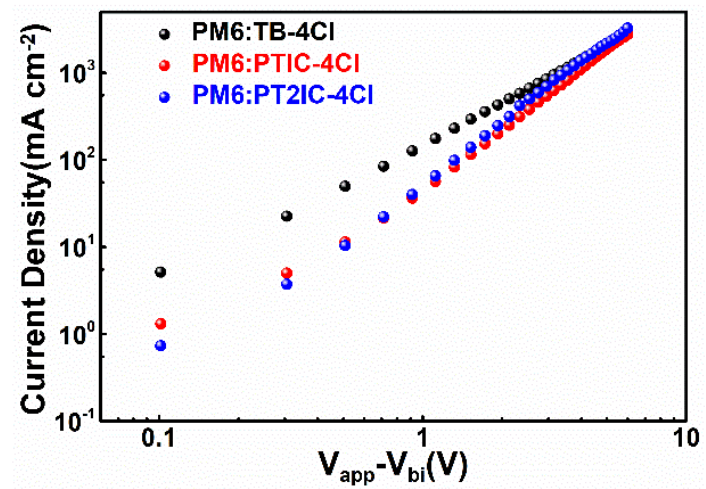

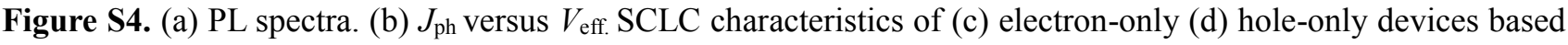
on PM6:TB-4Cl, PM6:PTIC-4Cl and PM6:PT2IC-4Cl blend films.

Table S1. The parameters of exciton dissociation and charge collection efficiency calculated from $J_{\mathrm{ph}}-V_{\text {eff }}$ curves.

\begin{tabular}{cccccc}
\hline Active layer & $J_{\mathrm{sat}}\left[\mathrm{mA} \mathrm{cm}^{-2}\right]$ & $J_{\mathrm{ph}^{\mathrm{a}}}\left[\mathrm{mA} \mathrm{cm}^{-2}\right]$ & $J_{\mathrm{ph}} \mathrm{b}\left[\mathrm{mA} \mathrm{cm}^{-2}\right]$ & $P_{\text {diss }}(\%)$ & $P_{\text {coll }}(\%)$ \\
\hline PM6: TB-4Cl & 23.81 & 23.20 & 20.85 & 97.44 & 87.56 \\
PM6: PTIC-4Cl & 23.94 & 23.30 & 20.82 & 97.32 & 86.97 \\
PM6:PT2IC-4Cl & 21.38 & 20.70 & 17.74 & 96.82 & 82.97 \\
\hline
\end{tabular}

${ }^{\mathrm{a}}$ Under short-circuit condition; ${ }^{\mathrm{b}}$ Under maximum power output. 


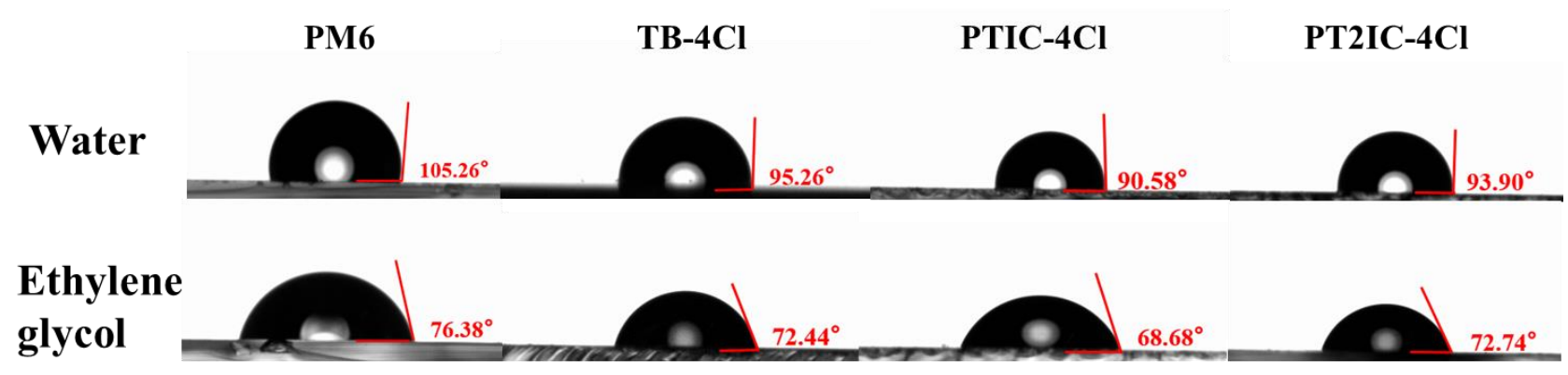

Figure S5. Contact angle images of water and ethylene glycol (EG) on PM6, TB-4Cl, PTIC-4Cl and PT2IC-4Cl neat films.

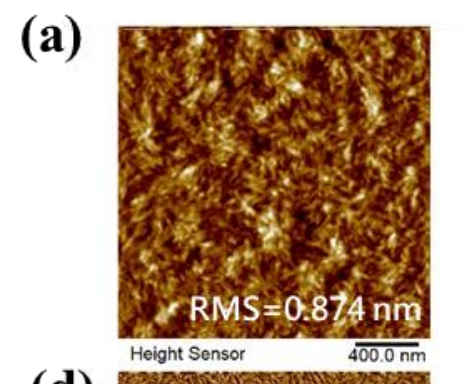

(d)

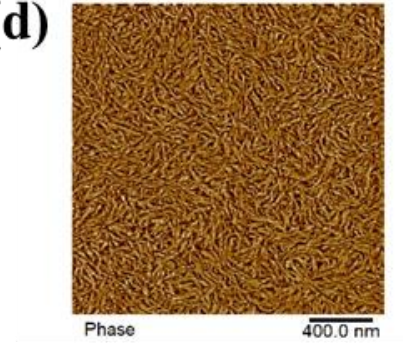

(b)

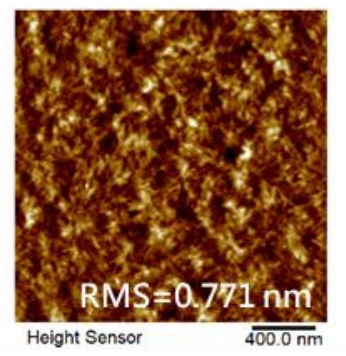

(e)

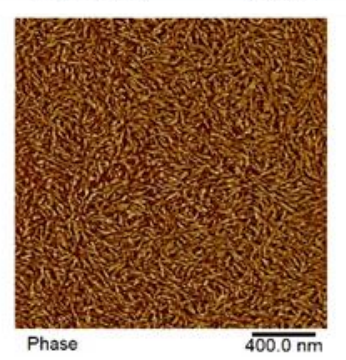

(c)

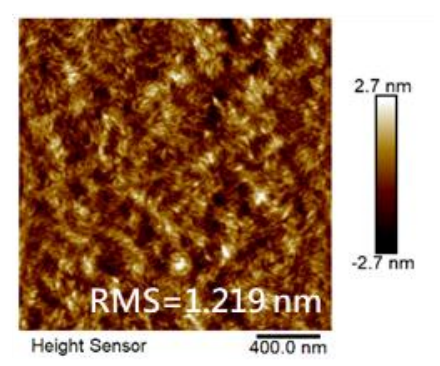

(f)

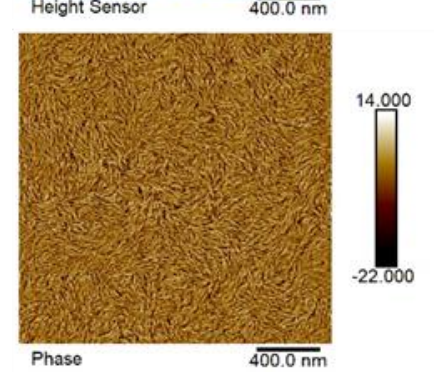

Figure S6. AFM height images (a)PM6: TB-4Cl, (b)PM6: PTIC-4Cl and (c)PM6: PT2IC-4Cl blend films and phase images of (d)PM6: TB-4Cl, (e)PM6: PTIC-4Cl and (f)PM6: PT2IC-4Cl blend films. 

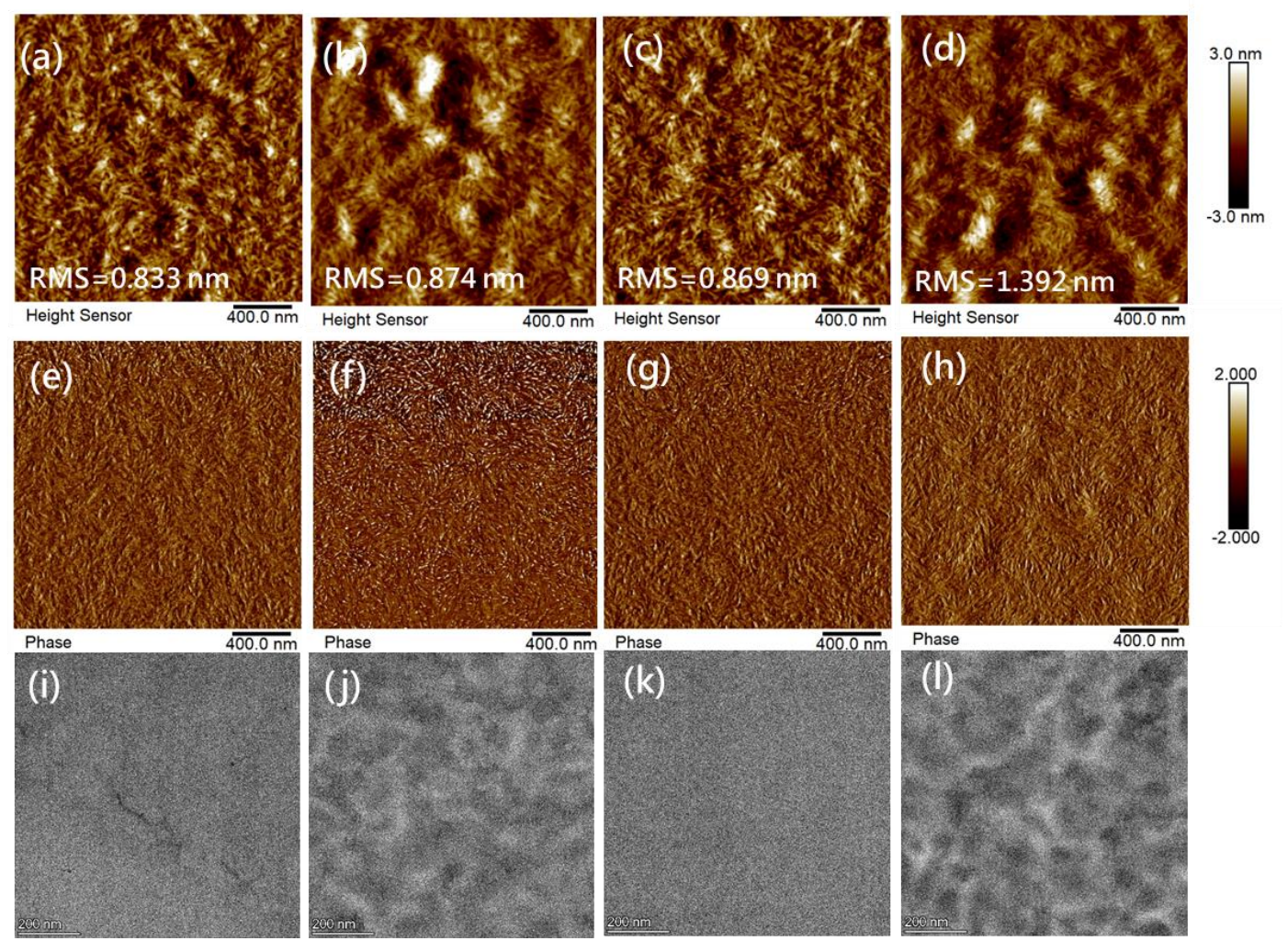

Figure S7 AFM height images (a-d), phase images (e-h) and TEM images (i-1). PM6:PTIC-4Cl blend films before (a, e, i) and after (b, f, j) annealing at $85^{\circ} \mathrm{C}$ for $140 \mathrm{~h}$; PM6:PTIC-4Cl blend films before (c, g, k) and after (d, h, l) storage in air for $180 \mathrm{~h}$.
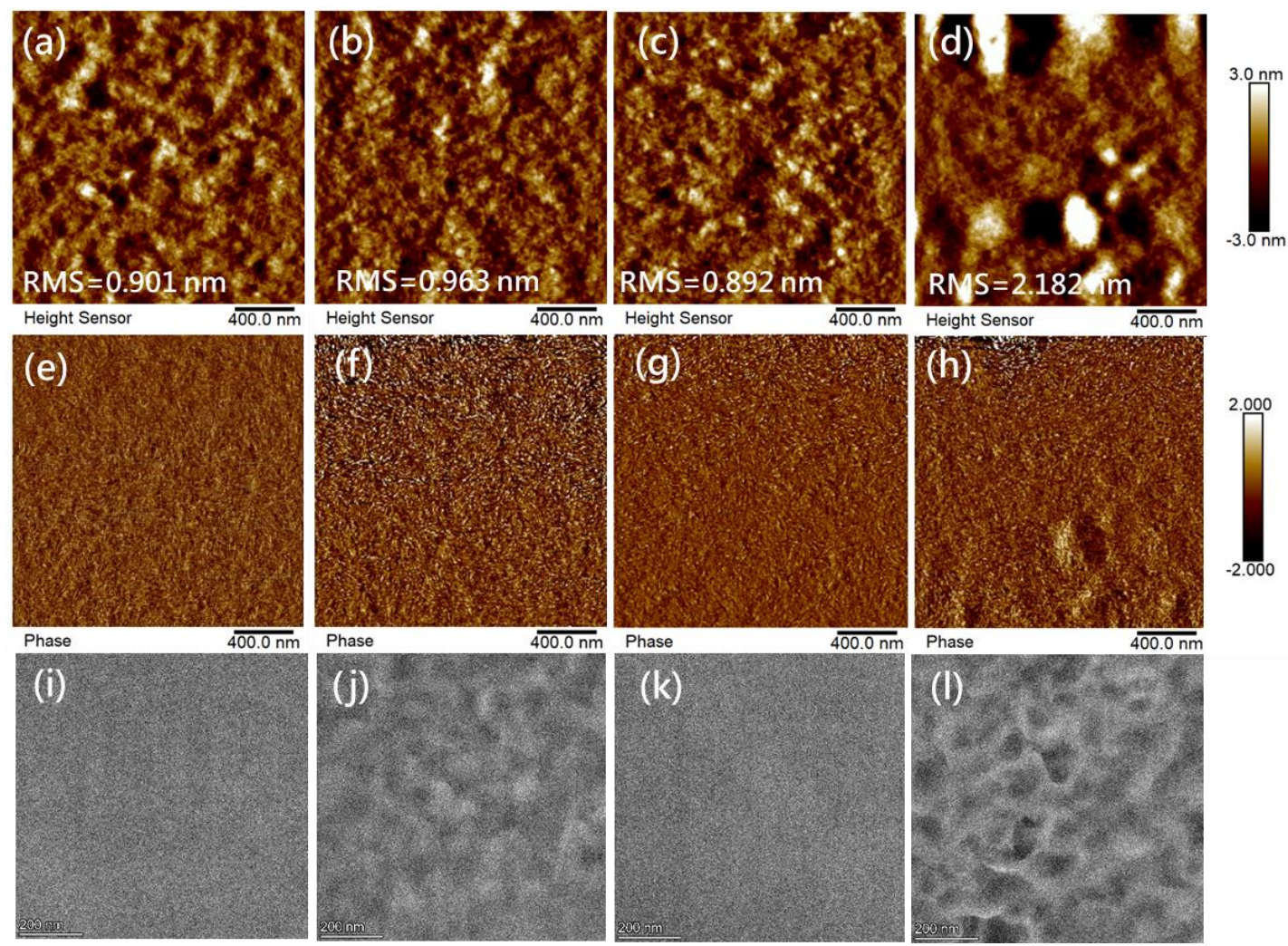

Figure S8 AFM height images (a-d), phase images (e-h) and TEM images (i-1). PM6:Y6 blend films before (a, e, i) and after (b, f, j) annealing at $85{ }^{\circ} \mathrm{C}$ for $140 \mathrm{~h}$; PM6:Y6 blend films before (c, g, k) and after (d, h, l) storage in air for $180 \mathrm{~h}$.. 
(a)

(b)
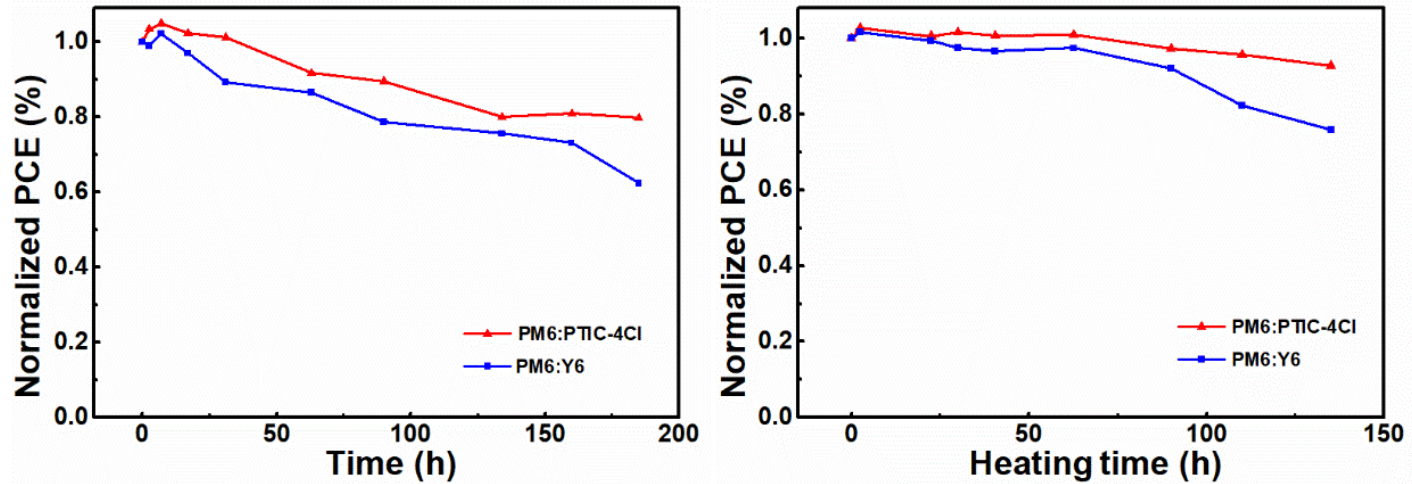

Figure S9. Normalized PCE of (a) air stability test. (b) Thermal stability test $\left(85^{\circ} \mathrm{C}\right.$ in nitrogen).

(a)

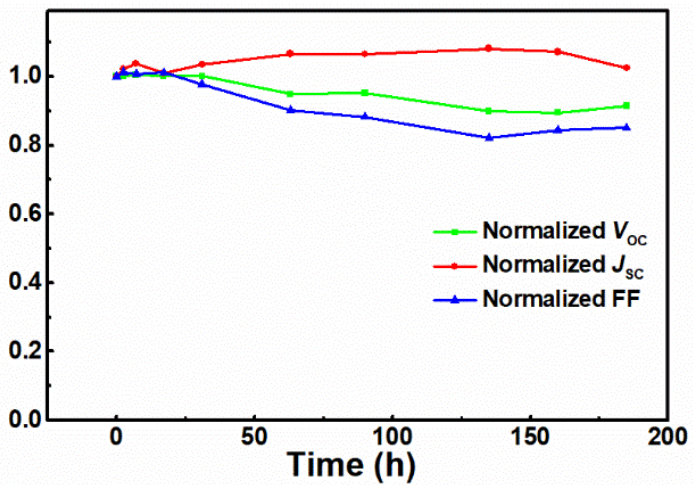

(b)

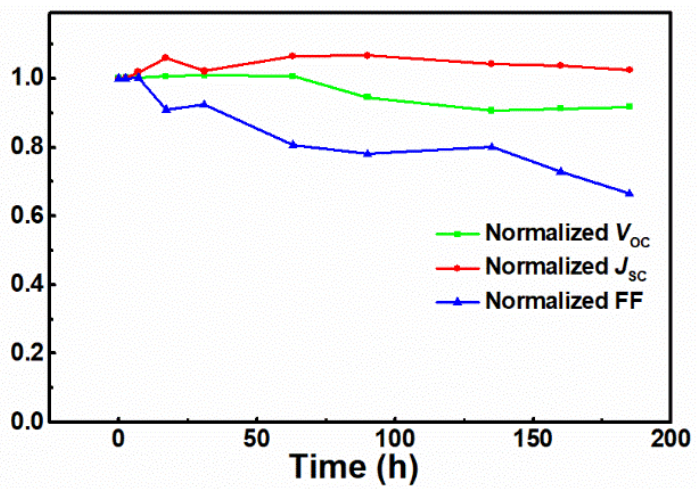

Figure S10. The photovoltaic parameters of air stability test (a) PM6:PTIC-4Cl. (b) PM6:Y6.

(a)

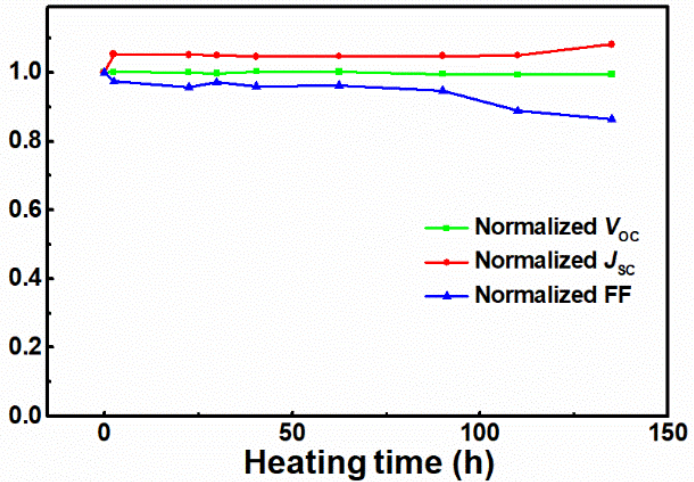

(b)

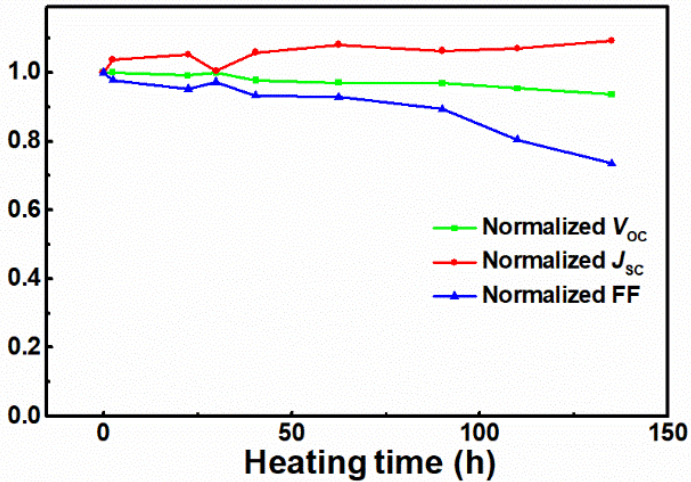

Figure S11. The photovoltaic parameters of thermal stability test $\left(85^{\circ} \mathrm{C}\right.$ in nitrogen) (a) PM6:PTIC-4Cl. (b) PM6:Y6. 


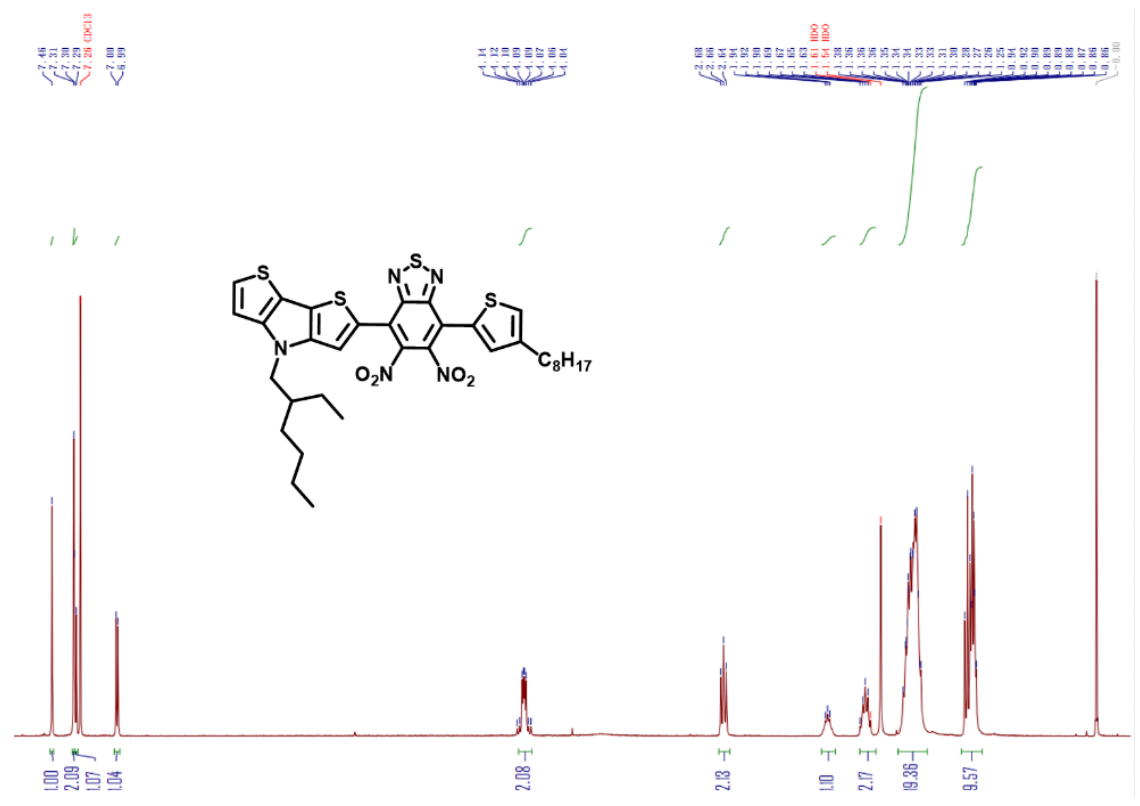

Figure S12. ${ }^{1} \mathrm{H}$ NMR spectrum of compound 4 in $\mathrm{CDCl}_{3}$.

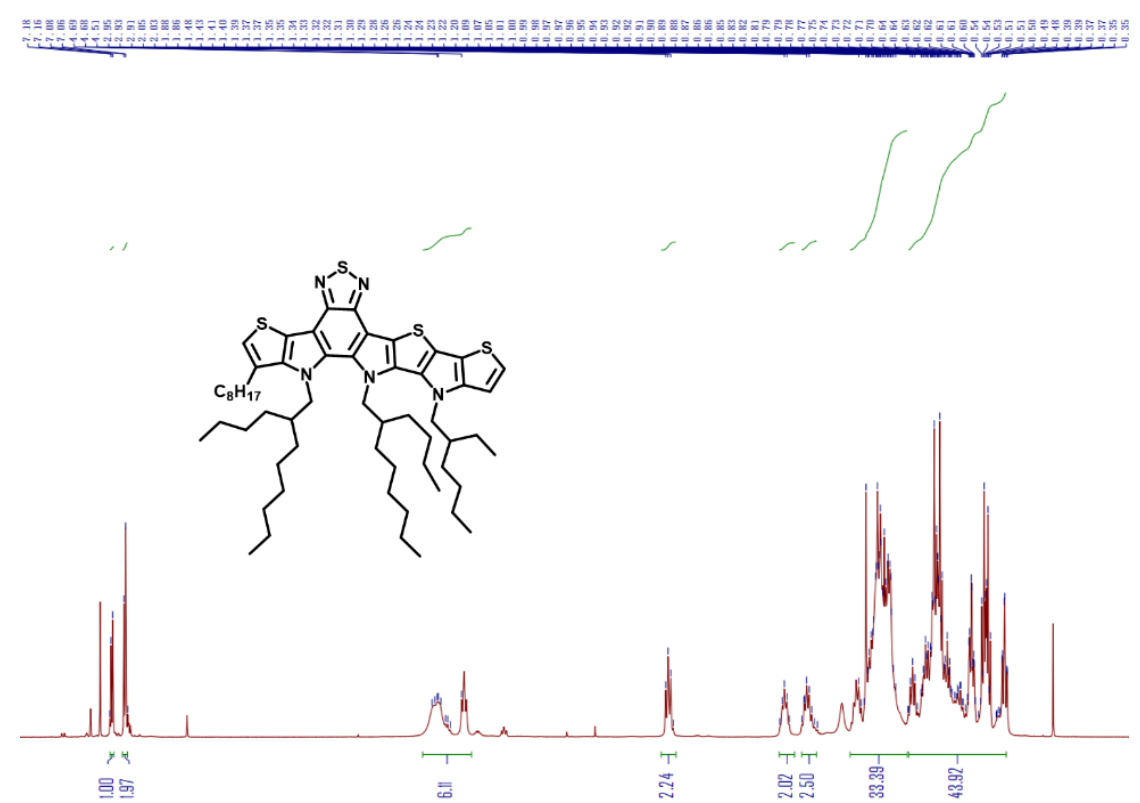

Figure S13. ${ }^{1} \mathrm{H}$ NMR spectrum of compound 5 in $\mathrm{CDCl}_{3}$. 


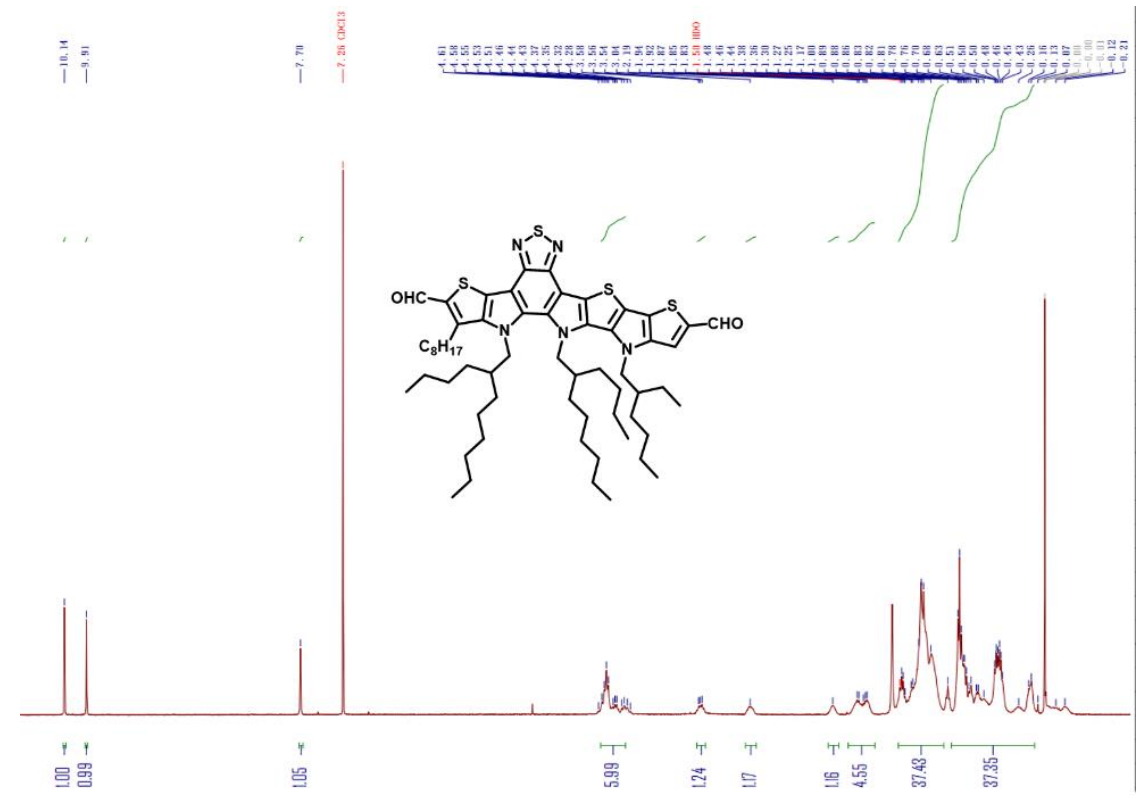

Figure S14. ${ }^{1} \mathrm{H}$ NMR spectrum of compound 6 in $\mathrm{CDCl}_{3}$.

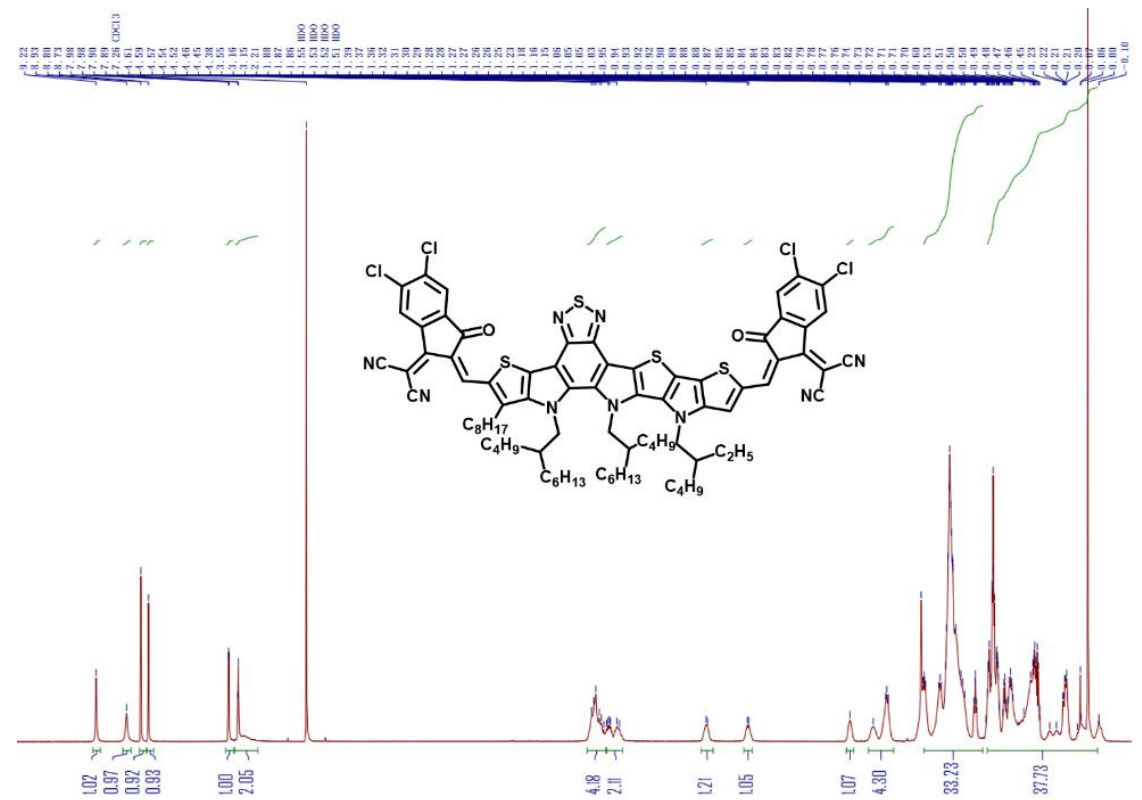

Figure S15. ${ }^{1} \mathrm{H}$ NMR spectrum of compound 7 in $\mathrm{CDCl}_{3}$. 


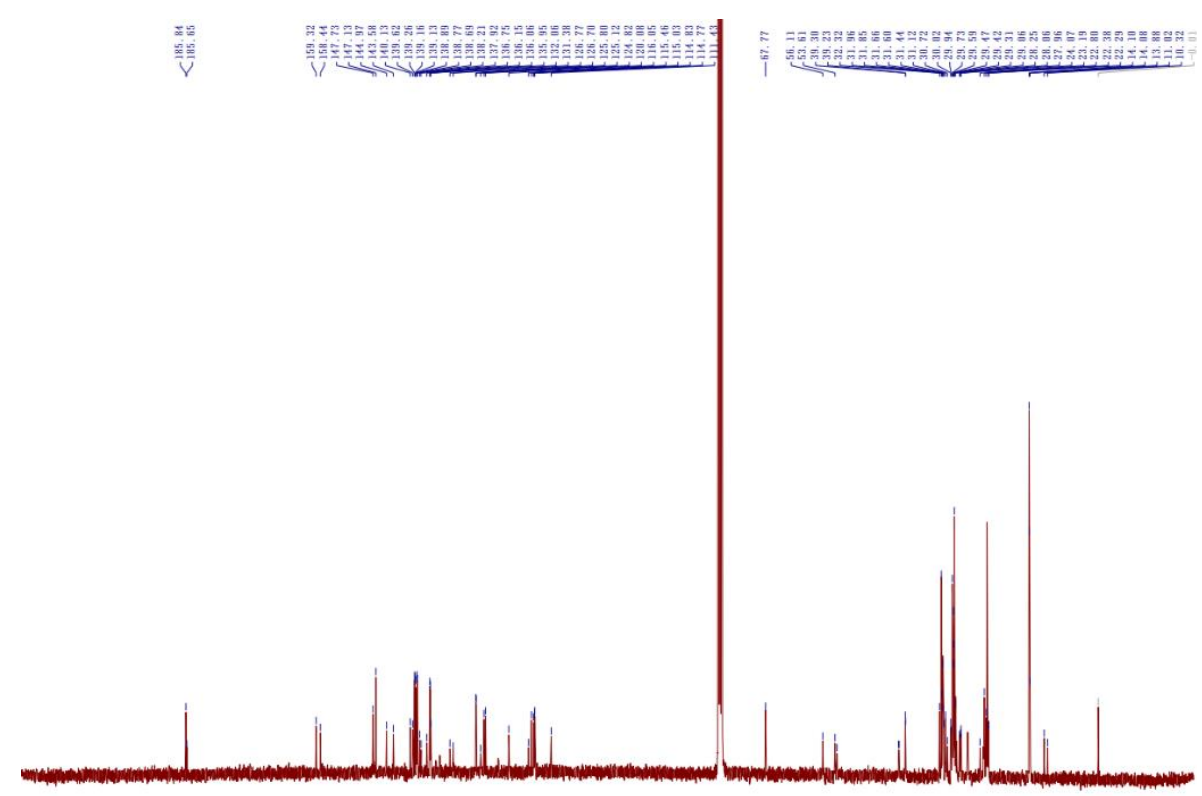

Figure S16. ${ }^{13} \mathrm{C}$ NMR spectrum of compound 7 in $\mathrm{CDCl}_{3}$.

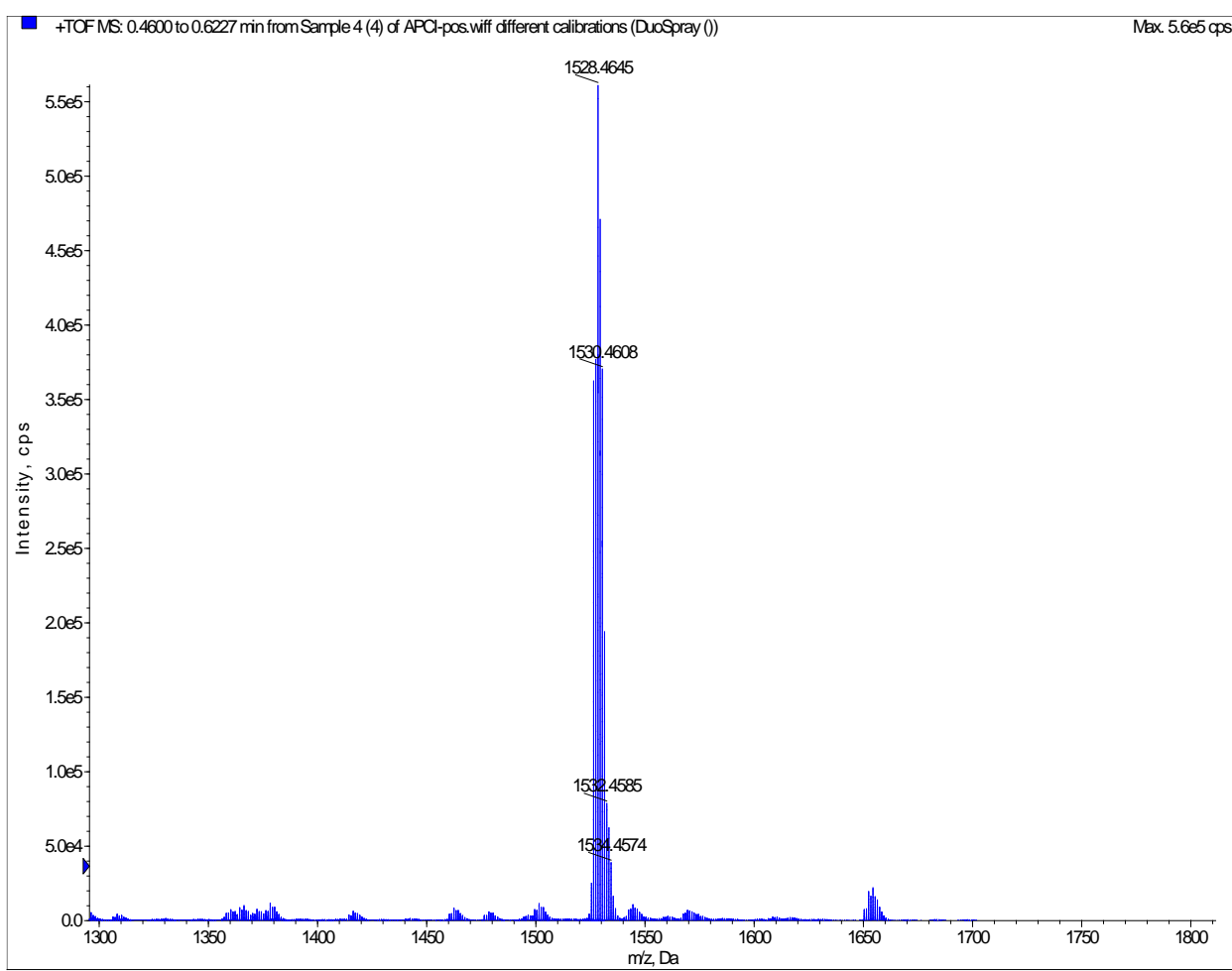

Figure S17. TOF spectrum of PTIC-4Cl. 


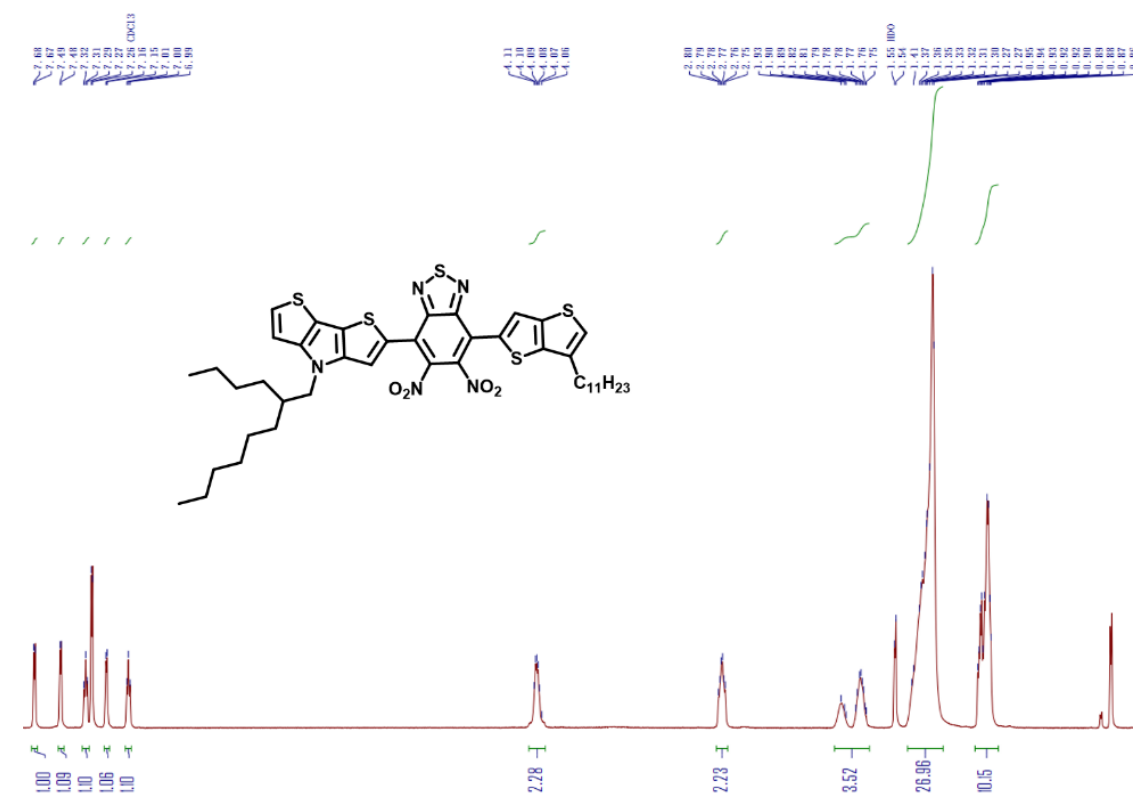

Figure S18. ${ }^{1} \mathrm{H}$ NMR spectrum of compound 9 in $\mathrm{CDCl}_{3}$.

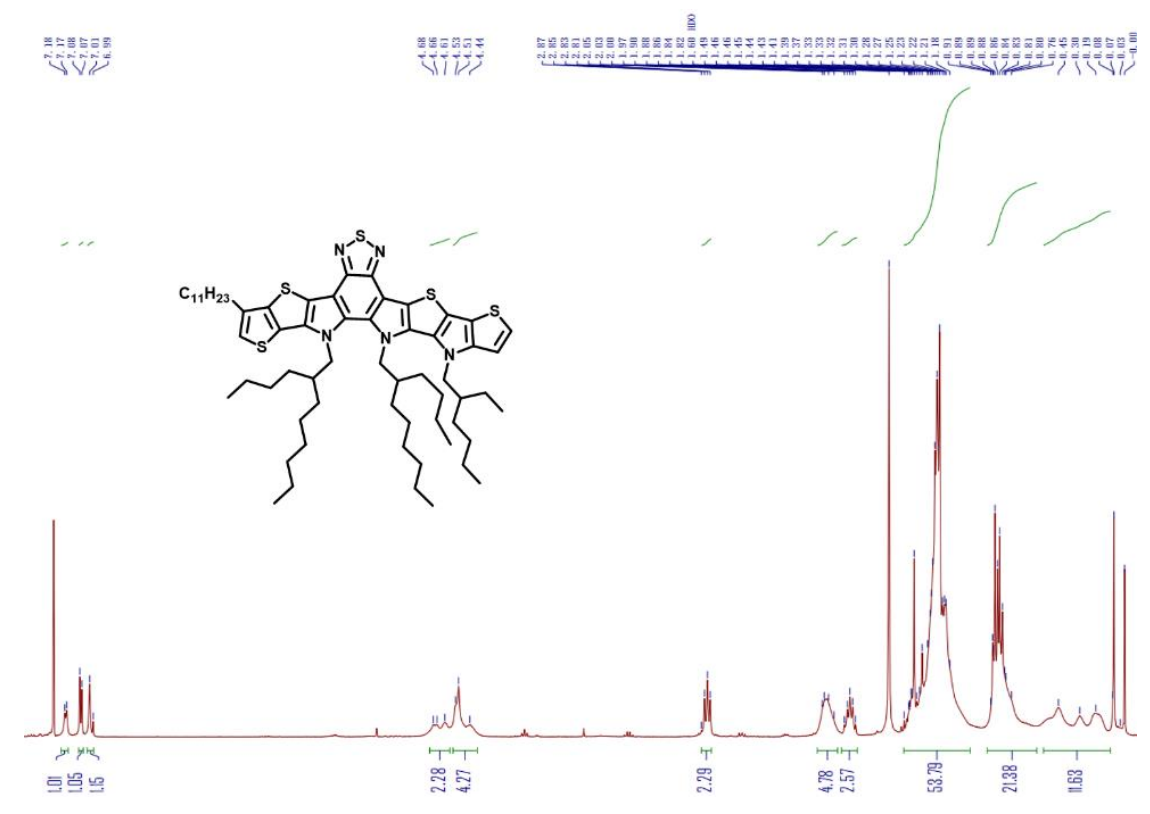

Figure S19. ${ }^{1} \mathrm{H}$ NMR spectrum of compound 10 in $\mathrm{CDCl}_{3}$. 


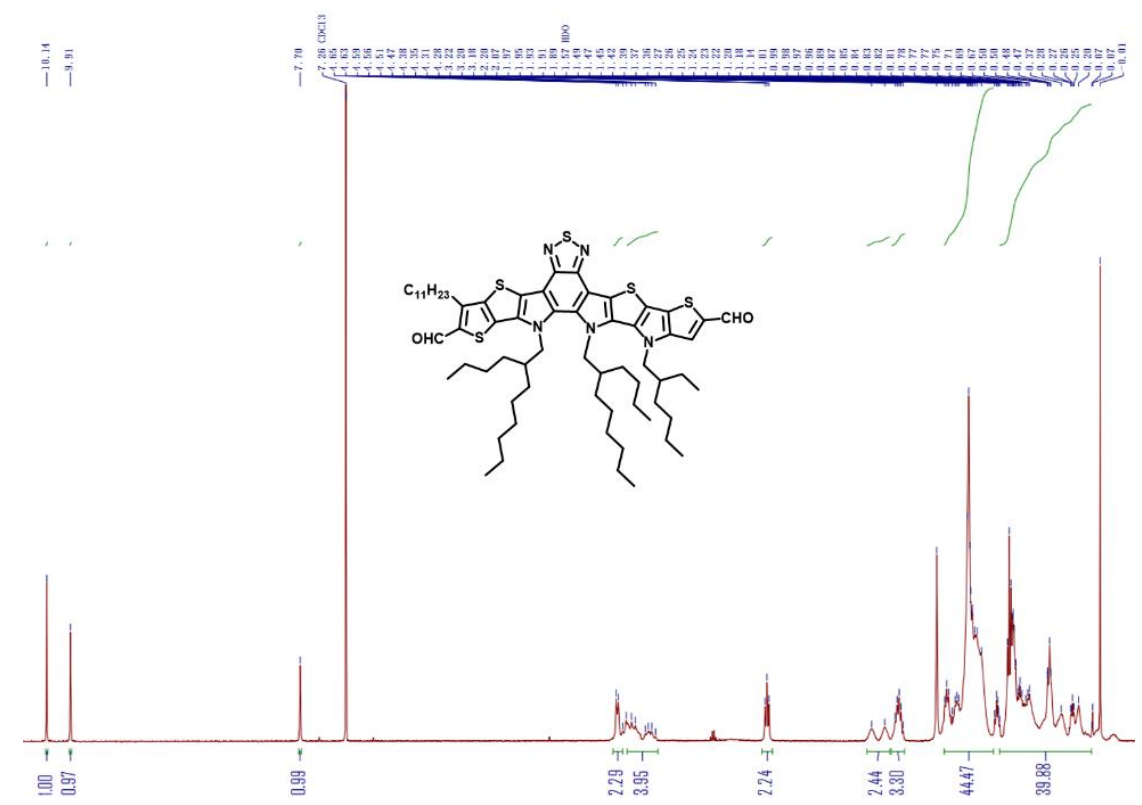

Figure S20. ${ }^{1} \mathrm{H}$ NMR spectrum of compound 11 in $\mathrm{CDCl}_{3}$.

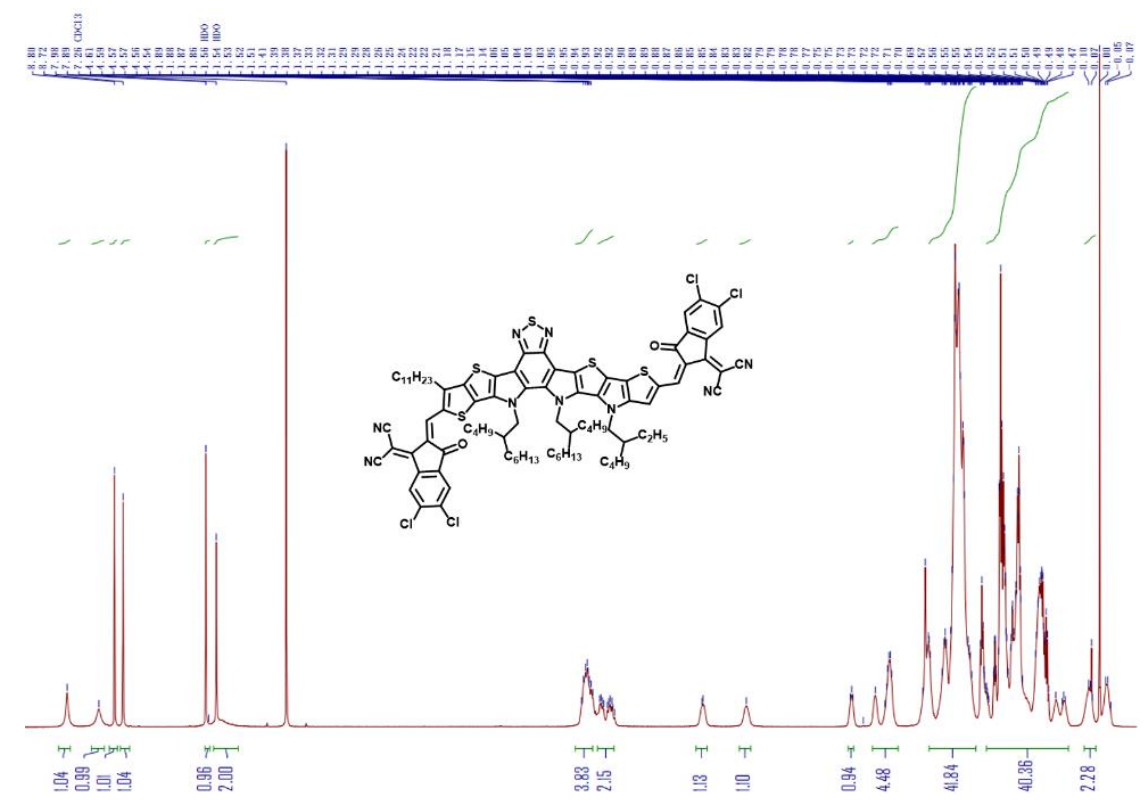

Figure S21. ${ }^{1} \mathrm{H}$ NMR spectrum of compound 12 in $\mathrm{CDCl}_{3}$. 


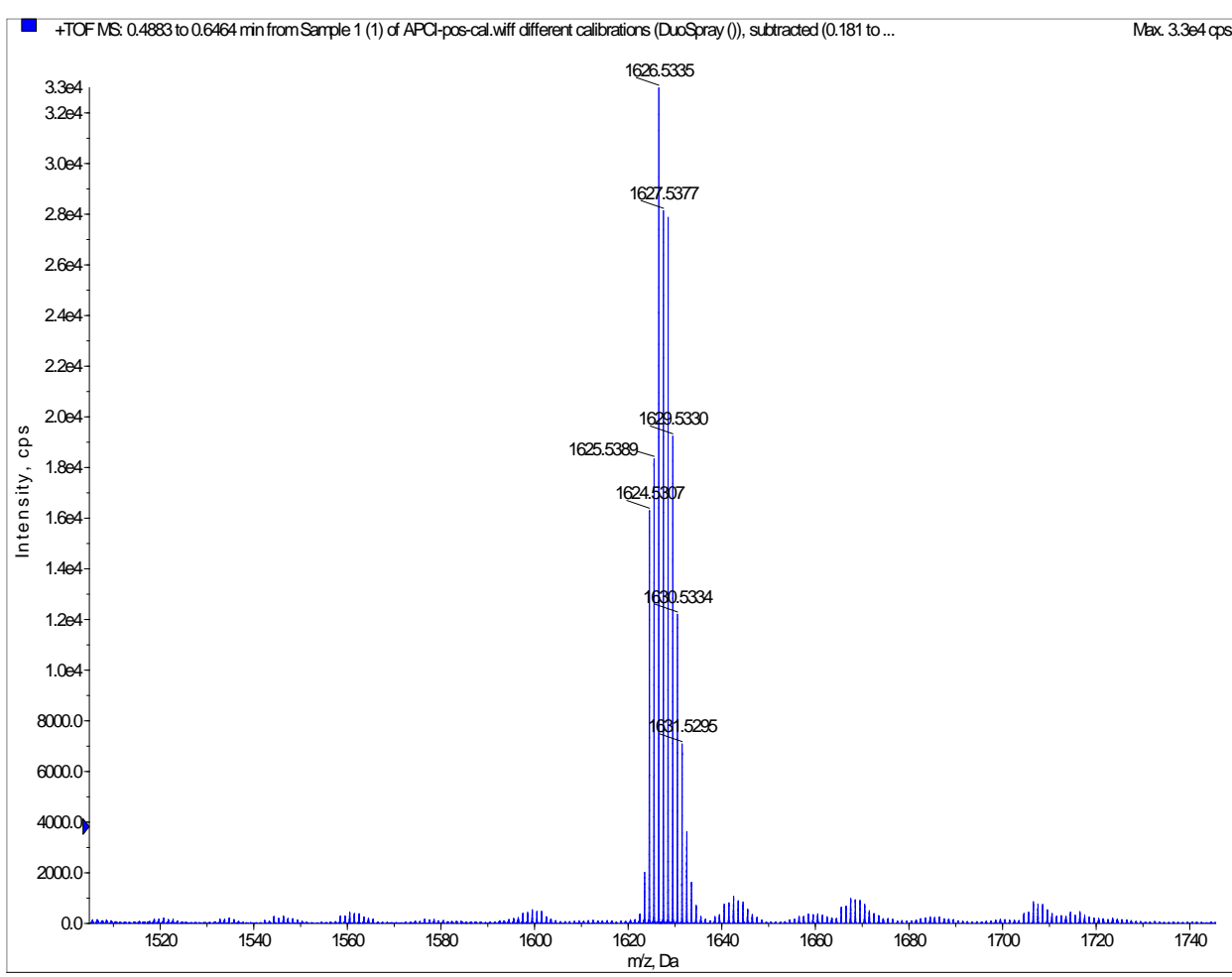

Figure S22. TOF spectrum of PT2IC-4Cl.

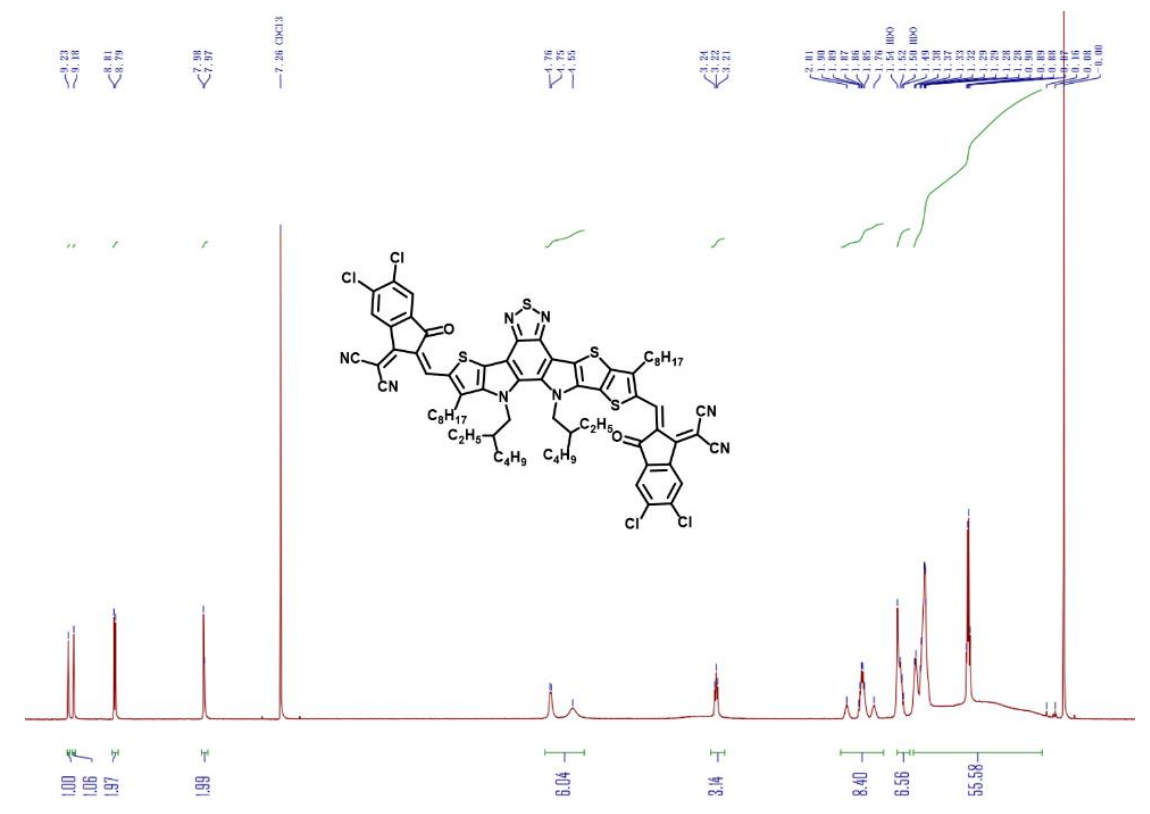

Figure S23. ${ }^{1} \mathrm{H}$ NMR spectrum of $\mathrm{TB}-4 \mathrm{Cl}$ in $\mathrm{CDCl}_{3}$. 


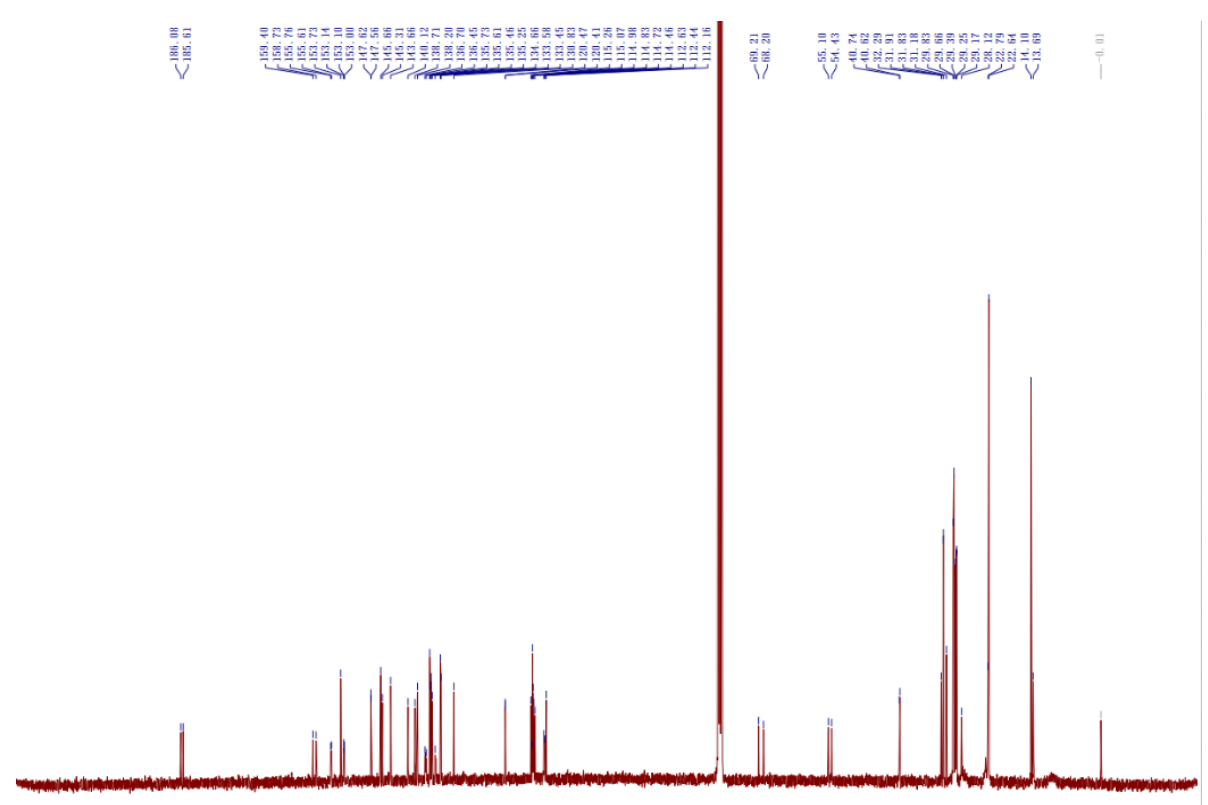

Figure S24. ${ }^{13} \mathrm{C}$ NMR spectrum of TB- $4 \mathrm{Cl}$ in $\mathrm{CDCl}_{3}$.

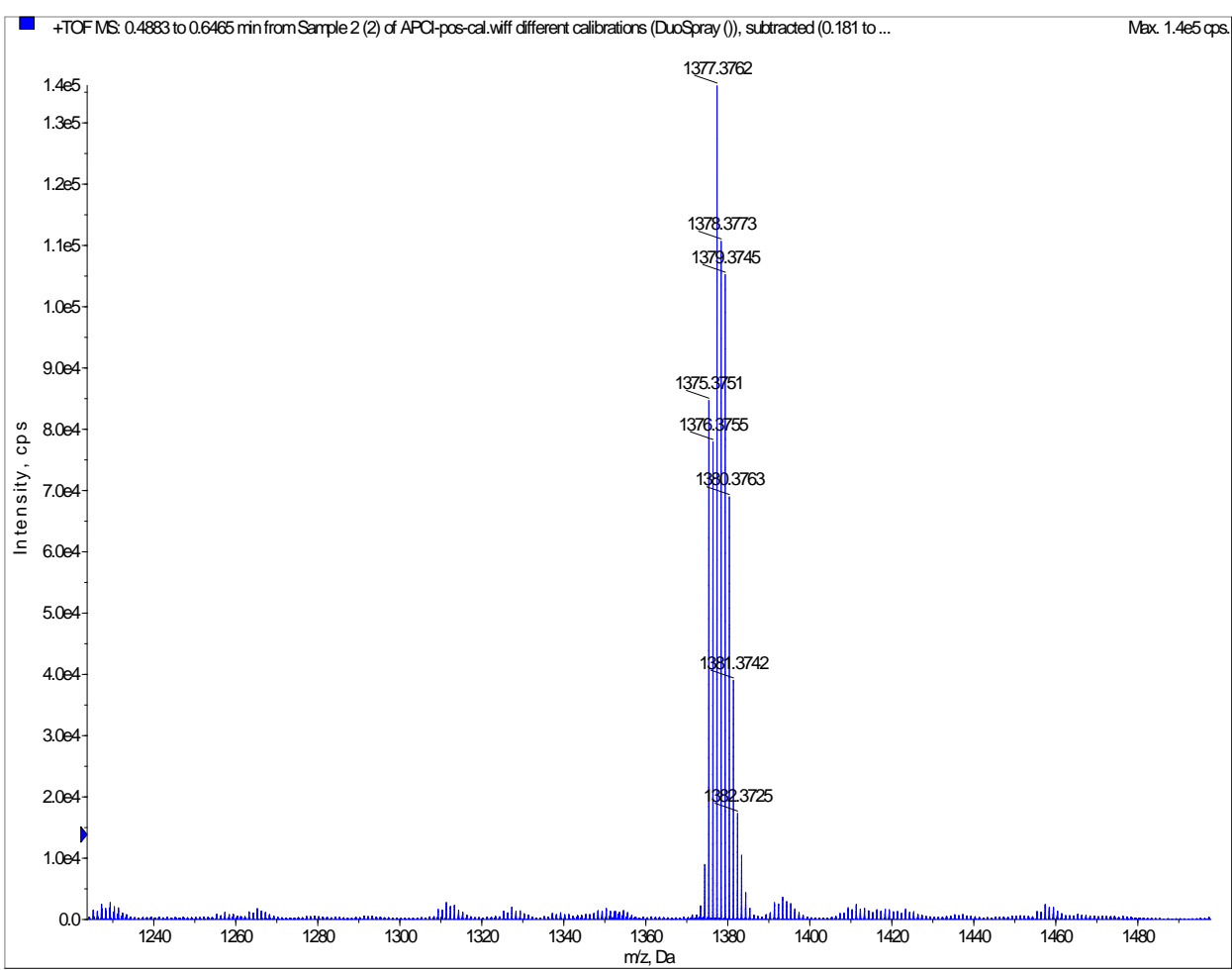

Figure S25. TOF spectrum of TB-4C1. 
Table S2. Photovoltaic performance of devices fabricated with PM6 and PTIC-4Cl with different concentration of additive.

\begin{tabular}{cccccc}
\hline Active layer & Additive & $J_{\mathrm{SC}}\left[\mathrm{mA} \mathrm{cm}^{-2}\right]$ & $V_{O C}[\mathrm{~V}]$ & FF [\%] & PCE (\%) \\
\hline & $0.2 \% 1-\mathrm{CN}$ & 22.71 & 0.863 & 71.62 & 14.02 \\
PM6:PTIC-4Cl & $0.5 \% 1-\mathrm{CN}$ & 23.31 & 0.860 & 73.90 & 14.76 \\
& $0.8 \% 1-\mathrm{CN}$ & 23.27 & 0.861 & 71.92 & 14.42 \\
& $1.0 \% 1-\mathrm{CN}$ & 22.38 & 0.863 & 71.07 & 13.74 \\
\hline
\end{tabular}

Table S3. Photovoltaic performance of devices fabricated with PM6 and PTIC-4Cl with different annealing temperatures.

\begin{tabular}{|c|c|c|c|c|c|}
\hline Active layer & Thermal annealing & $J_{\mathrm{SC}}\left[\mathrm{mA} \mathrm{cm}^{-2}\right]$ & $V_{O C}[\mathrm{~V}]$ & $\mathrm{FF}[\%]$ & PCE $(\%)$ \\
\hline \multirow{4}{*}{ PM6:PTIC-4Cl } & $80^{\circ} \mathrm{C}$ & 22.47 & 0.869 & 73.63 & 14.39 \\
\hline & $100^{\circ} \mathrm{C}$ & 22.81 & 0.866 & 74.28 & 14.68 \\
\hline & $120^{\circ} \mathrm{C}$ & 23.31 & 0.857 & 73.81 & 14.75 \\
\hline & $140^{\circ} \mathrm{C}$ & 23.49 & 0.856 & 71.70 & 14.47 \\
\hline
\end{tabular}

Table S4. Photovoltaic performance of devices fabricated with PM6 and PTIC-4Cl with various concentrations.

\begin{tabular}{cccccc}
\hline Active layer & Concentration & $J_{\mathrm{SC}}\left[\mathrm{mA} \mathrm{cm}^{-2}\right]$ & $V_{O C}[\mathrm{~V}]$ & FF [\%] & PCE (\%) \\
\hline & $14 \mathrm{mg} / \mathrm{ml}$ & 22.75 & 0.863 & 73.88 & 14.49 \\
PM6:PTIC-4Cl & $16 \mathrm{mg} / \mathrm{ml}$ & 22.87 & 0.862 & 74.40 & 14.67 \\
& $18 \mathrm{mg} / \mathrm{ml}$ & 23.30 & 0.860 & 73.89 & 14.80 \\
& $20 \mathrm{mg} / \mathrm{ml}$ & 22.42 & 0.862 & 73.49 & 14.21 \\
\hline
\end{tabular}

Table S5. Photovoltaic performance of devices fabricated with PM6 and SMAs with various D:A ratios.

\begin{tabular}{cccccc}
\hline Active layer & Blend ratio & $J_{\mathrm{SC}}\left[\mathrm{mA} \mathrm{cm}^{-2}\right]$ & $V_{O C}[\mathrm{~V}]$ & $\mathrm{FF}[\%]$ & PCE (\%) \\
\hline \multirow{3}{*}{ PM6:PTIC-4Cl } & $1: 1$ & 22.49 & 0.862 & 75.22 & 14.52 \\
& $1: 1.2$ & 23.25 & 0.862 & 73.93 & 14.82 \\
& $1: 1.4$ & 22.54 & 0.866 & 71.62 & 14.52 \\
\hline \multirow{3}{*}{ PM6:PT2IC-4Cl } & $1: 1$ & 19.71 & 0.836 & 72.12 & 11.90 \\
& $1: 1.2$ & 20.67 & 0.835 & 72.28 & 12.50 \\
& $1: 1.4$ & 20.65 & 0.838 & 71.65 & 12.41 \\
\hline \multirow{3}{*}{ PM6:TB-4Cl } & $1: 1$ & 22.89 & 0.812 & 73.19 & 13.70 \\
& $1: 1.2$ & 23.19 & 0.835 & 74.49 & 14.42 \\
& $1: 1.4$ & 22.56 & 0.847 & 73.12 & 14.22 \\
\hline
\end{tabular}




\section{Reference}

(1) Zhang, J.; Han, Y.; Zhang, W.; Ge, J.; Xie, L.; Xia, Z.; Song, W.; Yang, D.; Zhang, X.; Ge, Z., High-Efficiency Thermal-Annealing-Free Organic Solar Cells Based on an Asymmetric Acceptor with Improved Thermal and Air Stability. ACS Appl Mater Interfaces. 2020, 12 (51), 57271-57280. 